\title{
Final Scientific and Technical Report
}

DOE award \#: DE-SC0001821

Recipient: $\quad$ T2 Biosystems, Inc.

286 Cardinal Medeiros Ave

Cambridge, MA 02141

Project Title: Development of a System for Rapid Detection of Contaminants in Water Supplies Using Magnetic Resonance and Nanoparticles

PI: $\quad$ Thomas J Lowery

Team: $\quad$ Thomas J Lowery, Lori Neely, James Chepin, Parris Wellman, Ken Toso, Paul Murray, Mark Audeh, Vasiliki Demas, Robert Palazzolo, Michael Min, Nu Phung, Matt Blanco, Jordan Raphel, Troy O’Neil

\section{Table of Contents}

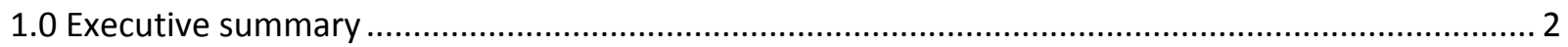

2.0 Performance against Goals and Objectives ............................................................................ 2

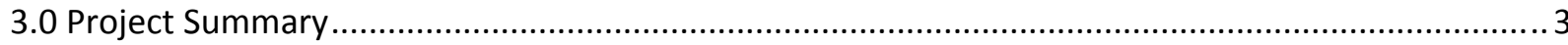

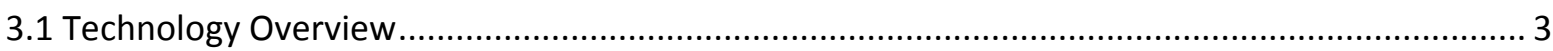

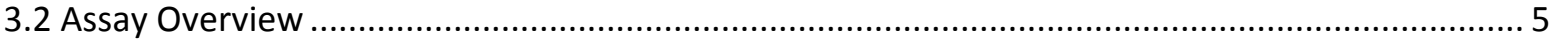



3.4 Portable Magnetic Resonance Detection Device....................................................................... 7

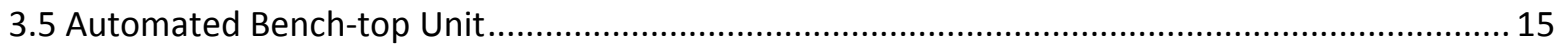

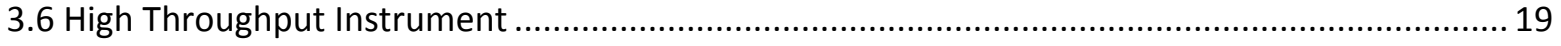

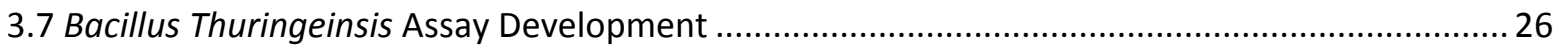

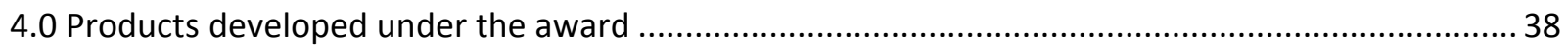

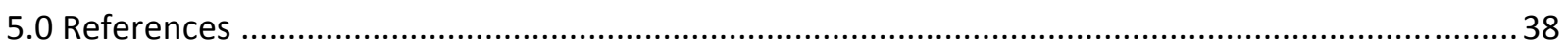


Final Scientific \& Technical Report: Development of a System for Rapid Detection of Contaminants in Water Supplies Using Magnetic Resonance and Nanoparticles

\subsection{Executive summary}

To keep the water supply safe and to ensure a swift and accurate response to a water supply contamination event, rapid and robust methods for microbial testing are necessary. Current technologies are complex, lengthy and costly and there is a need for rapid, reliable, and precise approaches that can readily address this fundamental security and safety issue. T2 Biosystems is focused on providing solutions to this problem by making breakthroughs in nanotechnology and biosensor techniques that address the current technical restrictions facing rapid, molecular analysis in complex samples. In order to apply the T2 Biosystems nucleic acid detection procedure to the analysis of nucleic acid targets in unprocessed water samples, Bacillus thuringeinsis was selected as a model organism and local river water was selected as the sample matrix. The initial assay reagent formulation was conceived with a manual magnetic resonance reader, was optimized using a high throughput system, and transferred back to the MR reader for potential field use. The final assay employing the designed and manufactured instruments was capable of detecting $10 \mathrm{CFU} / \mathrm{mL}$ of $B$. thuringiensis directly within the environmental water sample within 90 minutes. Further, discrimination of two closely related species of Bacilli was accomplished using the methods of this project; greater than 3-fold discrimination between $B$. cereus and $B$. thuringiensis at a concentrations spanning $10 \mathrm{CFU} / \mathrm{mL}$ to $10^{5} \mathrm{CFU} / \mathrm{mL}$ was observed.

\subsection{Performance against Goals and Objectives}

T2 Biosystems (T2Bio) has achieved the goals and objectives of DOE project DE-SC0001821. As set out in the original proposal, the goals of this project were the following:

Goal 1: Develop a qualified test for the detection of a microbial pathogen that validates that an MR system can detect the presence of pathogens in water samples.

Goal 2: Develop a prototype MR instrument that can be used for the detection of a microbial pathogen in water samples.

To achieve these goals, the project was approached from T2Bio's integrated engineering and assay development programs, and the corresponding objectives were the following:

Objective 1: Engineer a portable magnetic resonance detection device to robustly measure $\mathbf{T}_{\mathbf{2}}$ relaxation time. The system was an all-in-one design, incorporating a permanent magnet, computer, and spectrometer components into a single, compact and ergonomic enclosure for use with specifically designed assays, as in this funded project, pathogen detection in contaminated water.

Objective 2: Develop reagents and assay formats that optimize analysis from unprocessed water samples. Nucleic acid targets of a specific pathogen were identified and detected using magnetic relaxation switches in contaminated water.

As described below, all of the goals and objectives of the project were met. As partial fulfillment of this project, several data sets have been collected on the portable bench top magnetic resonance unit 
Final Scientific \& Technical Report: Development of a System for Rapid Detection of Contaminants in Water Supplies Using Magnetic Resonance and Nanoparticles

demonstrating the capability of detecting Bacillus thuriengensis in a variety of matrices, including river water.

\subsection{Project Summary}

T2Bio is developing rapid, point-of-need biological sensors based on nanotechnology and magnetic resonance detection. In this project, T2Bio has developed a microbial testing assay to be used on a previously designed diagnostic platform for rapid detection of contaminants in water. The specific project hypothesis was that a magnetic resonance detection platform utilizing superparamagnetic nanoparticles can be engineered into a portable device capable of performing nucleic acid detection on minimally processed environmental water samples. That hypothesis was based on the following observations. First, magnetic resonance detection technology (boards, spectrometer, magnets, and radio frequency coils) can be miniaturized to fit into a portable system while maintaining high sensitivity and precision. Second, superparamagnetic nanoparticles can serve as magnetic relaxation switches when they react with multiple types of molecular targets to undergo changes in aggregation state and thereby affect solvent magnetic resonance relaxation. Third, nanotechnology-mediated magnetic detection devices can be engineered to work in minimally processed, 'dirty' or heterogeneous samples such as untreated water. Based on these observations, the focus of this project was on the use of magnetic relaxation switches (MRSw) as a novel diagnostic nuclear magnetic resonance (NMR) platform. The specific aims are to comprehensively examine and develop MRSw measurements as a portable detection method for use in detecting the pathogens in river water, demonstrate the platform's capability by employing as yet an untested method to detect pathogens in dirty water samples, while presenting innovative solutions to the problem of rapid and cost efficient detection of water supply contamination.

\subsection{Technology Overview}

T2Bio designed diagnostic platform utilizes portable magnetic resonance and superparamagnetic nanoparticle technologies to afford rapid and simple detection of biological targets in complex matrices. No existing diagnostic instruments are capable of direct amplification and detection of nucleic acids without significant sample preparation steps. Innovative solutions are needed to simplify the analytic process and provide a cost-effective method that can be applied broadly. By uniting the principles of $T_{2}$ detection and nanotechnology, a multifunctional and multi-analyte diagnostic system satisfies this need. Specifically with nanotechnology, the phenomenon of superparamagnetism that occurs in nanoscale iron oxide crystals can be exploited as a unique diagnostic tool. Nanoparticles impregnated with these crystals have been used to create a portable, rapid, diagnostic system that enables advantages such as eliminating the need for sample preparation and the ability to use a simple, portable detector. Superparamagnetic nanoparticles serve as MRSw when they react with a specific biomolecular target to undergo changes in their aggregation state and thereby affect solvent $\mathrm{T}_{2}$ relaxation. These nanotechnology-mediated magnetic detection devices are engineered to work directly in minimallyprocessed samples such as blood or river water that optical systems cannot analyze. 
This highly sensitive nucleic acid detection platform circumvents the need for typical sample preparation to provide very rapid turn-around times at low complexity and low cost. The attributes of the T2Bio nucleic acid detection system include simplified sample processing, high sensitivity, rapid turn-around time, low cost, multiplexing, and multiple analyte detection capabilities (i.e. immunoassays can be run on the same sample, cartridge, and instrument). All of these features combine to create a simple-to-use, cost-effective system.

The physical property used for detection is $T_{2}$ (transverse or spin-spin) relaxation time, a measure of how long protons remain synchronized during a series of $180^{\circ}$ radio frequency (RF) pulses. The $T_{2}$ relaxation signal of naturally occurring water within any fluid sample (e.g. river water, whole blood, plasma, urine, etc) is measured by a MR detector, which consists of a small permanent magnet and radiofrequency coil as illustrated in Figure 1 . Self-assembly of superparamagnetic nanoparticles cause significant changes in $T_{2}$ relaxation times of surrounding water molecules which can be measured directly with the MR reader, as shown pictorially in Figure 2. The agglutination state of superparamagnetic particle reagents is the primary factor that dictates the $T_{2}$ relaxation signal. The nanoparticle clustering reactions can be induced by the presence of specific analytes (proteins, nucleic acid, viral particles etc.) and the resulting data indicates strong correlation between the measured $T_{2}$ values and the analyte concentration, which has been used for qualitative and quantitative measurements of analytes (Lowery 2008).

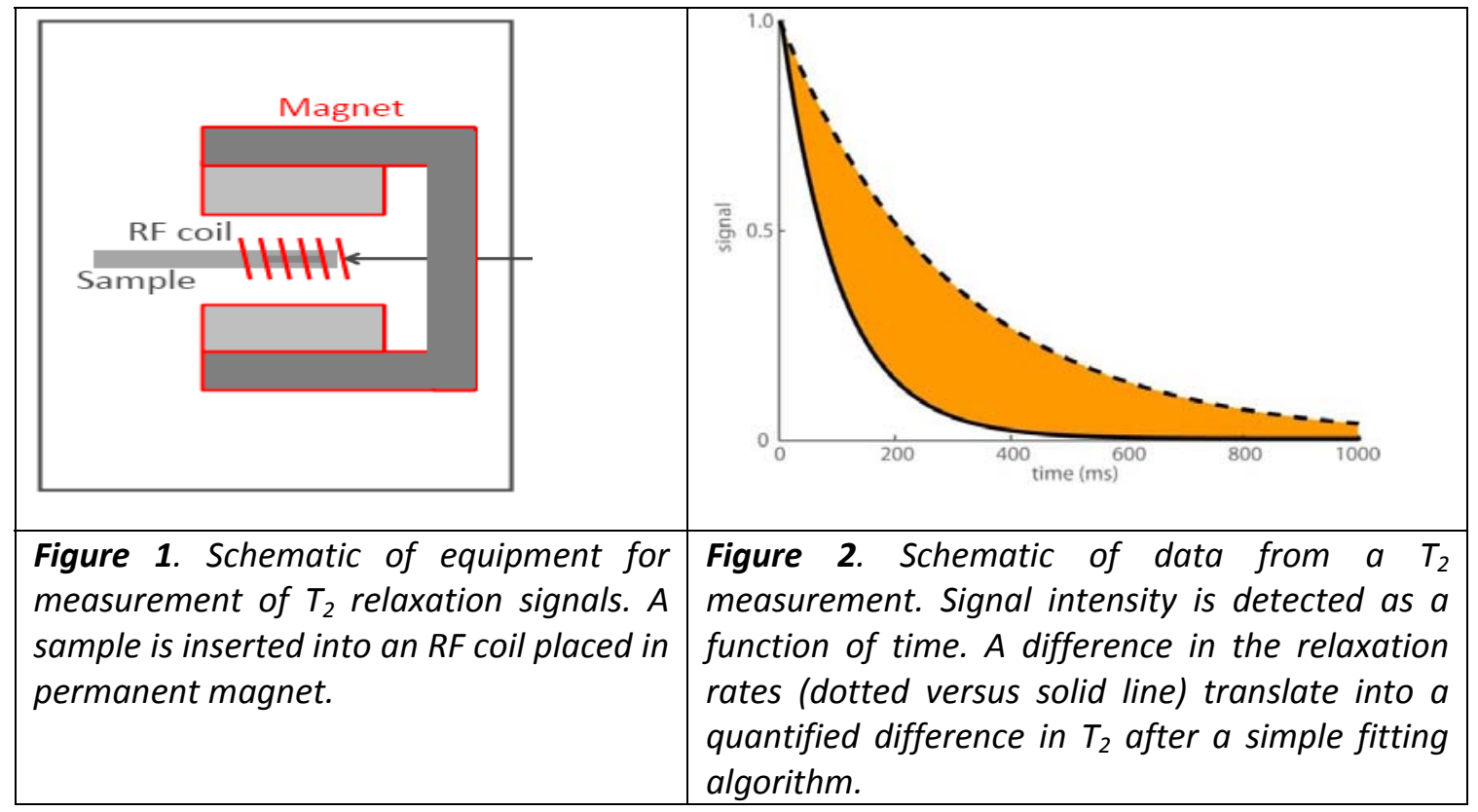

In the MRSw assays developed at T2Bio for nucleic acid analysis, conventional PCR primers and capture probes are used with the T2Bio technology to afford direct amplification and detection within the sample matrix. This is achieved by coupling the broad sample compatibility of the T2Bio detector with a proprietary enzyme reagent that can conduct PCR amplification in an unprocessed matrix like river water with otherwise standard reagents. The insensitivity of this enzyme to common inhibitors 
abrogates the need for the extensive sample preparation and purification. The lack of extensive sample manipulation limits variability introduced through sample preparation and eliminates the risk of target loss. After lysis treatment, the target pathogen nucleic acids are amplified via thermocycling directly within the sample matrix. After amplification, an aliquot of the sample is mixed with a solution of superparamagnetic particles. This solution consists of two populations of nanoparticles, each of which are conjugated to a capture probe complementary to the ends of the amplicon, as shown in Figure 3. The amplicon therefore serves as an interparticle bridge, promoting nanoparticle agglomeration. The number of nanoparticle bridges (amplicons) correlates with the presence of pathogenic DNA in the sample. After incubation of the sample with the particle solution to allow the amplicon to hybridize to the capture probes the amount of aggregation is detected via a $T_{2}$ measurement directly in the sample matrix.



Figure 3: A homogenous assay for detection of target nucleic acids. Oligonucleotide probes are covalently conjugated to super paramagnetic nanoparticles. For each target, two populations of nanoparticles are used, each bearing a distinct target complementary probe. Upon hybridization to the sense strand of the target, nanoparticle clustering is induced with the extent of clustering increasing concomitantly with the target DNA concentration.

\subsection{Assay Overview}

T2Bio's molecular diagnostics (MolDx) assay that enables high sensitivity detection of pathogens in unprocessed samples was originally developed on a whole blood matrix for a fungal target. This work produced and validated this assay format and demonstrated the following performance specifications: a diagnostic sensitivity and specificity of $>95 \%$ at target concentrations as low as $10 \mathrm{CFU} / \mathrm{mL}, 100 \%$ diagnostic accuracy as compared to blood culture reference method for the detection of clinical fungal isolates spiked into whole blood, $0.8 \mathrm{~mL}$ sample volumes, and a turn around time of between 90-150 minutes.

These assay performance metrics were achieved by combining T2Bio's dirty-sample compatible magnetic resonance based detector, superparamagnetic particles, and dirty-sample amplification 
Final Scientific \& Technical Report: Development of a System for Rapid Detection of Contaminants in Water Supplies Using Magnetic Resonance and Nanoparticles

technology into a single, straightforward assay workflow, as depicted in Figure 4. This assay workflow is much simpler than current existing methods that require purification of extracted nucleic acid prior to amplification and detection.

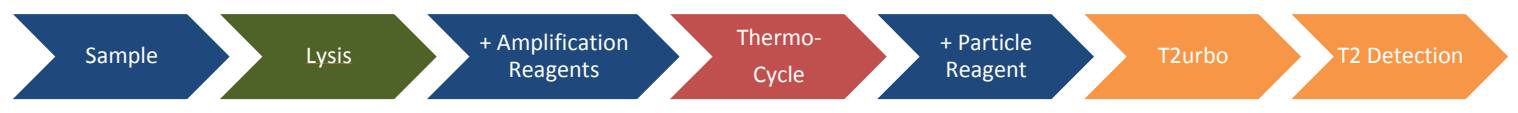

Figure 4. Assay workflow for T2Bio's molecular diagnostic assay. Fluidic handling steps are colored blue, lysis procedure green, thermocylcing red, and T2Bio's acceleration and $\mathrm{T}_{2}$ detection steps orange.

\subsection{Instrument Overview}

The initial data supporting T2Bio's MolDx assay described above was collected with a commercially available magnetic resonance (MR) relaxometer, the Bruker Minispec, and a T2Bio prototype magnetic resonance detector that consisted of a spectrometer, magnet, and accompanying detection circuitry inside of a temperature controlled enclosure. This detector was completed as part of T2Bio's immunoassay development work.

T2Bio hypothesized that this prototype could be utilized in two different use scenarios: (1) manual sample processing with a small portable instrument, and (2) automated processing on a portable bench top unit. The rationale behind this hypothesis was that a small portable instrument that allowed manual assay processing would be flexible during assay development and allow for portable applications that require processing of test samples at limited throughput. Additionally, the fully automated instrument was expected to allow for reduced user interaction with the system and increased assay throughput.

These two expanded prototypes were completed and testing commenced in the first four months of this DOE project and were evaluated for viability in MolDx assay development and processing. From this initial testing and development it was concluded that the portable MR reader was useful for assay development and manual processing of samples at throughputs of $<200$ measurements/day. The prototype fully automated bench top system that combined the automatic fluidic handling with the MR reader was optimal for hands-free assay processing, but less suited for modifying and optimizing assay workflows and did not provide adequate throughput needed by a day-to-day assay development platform. Accordingly, efforts were focused on the further development of the MR reader, which could provide a robust detector for all embodiments of instrumentation.

In order to address the need for higher throughput, it was decided to modify the existing prototype high throughput (HTP) system developed for immunoassays for the molecular assay. The HTP system had been demonstrated to work with a prototype MR reader but had not yet been used for MolDx assays It was hypothesized that the HTP system could significantly accelerate the reagents optimization process T2 Biosystems 
Final Scientific \& Technical Report: Development of a System for Rapid Detection of Contaminants in Water Supplies Using Magnetic Resonance and Nanoparticles

that was critical for successful assay design. Ideally, the initial assay reagent formulation would be conceived with the manual MR reader, then optimized using the HTP system, and transferred back to the MR reader for field use. The final format of the instrument components for executing the Bacillus thuringiensis assay workflow was envisioned to be modular, consisting of an MR reader, thermocycling unit, cartridge with removable tubes, T2urbo assay accelerator stations and an incubation station.

\subsection{Portable Magnetic Resonance Detection Device}

All three use scenarios (stand-alone manual system, automated system with integrated fluidics, high throughput system) considered during this project required a robust $\mathrm{T}_{2}$ relaxometer. Previous work at T2Bio had shown the feasibility of integrating all of the components for a $T_{2}$ detector into a single instrument. While each of the earlier embodiments of the $T 2$ Readers ( $T_{2}$ relaxometers) from the first demonstration of a small benchtop system that wasn't temperature controlled to the first temperature controlled breadboard Reader built in FY Q3 2009 (Figure 5) were stepwise improvements in performance and cost, some improvements still remained to be made to enable a robust, reliable T2 Reader for routine assay development.

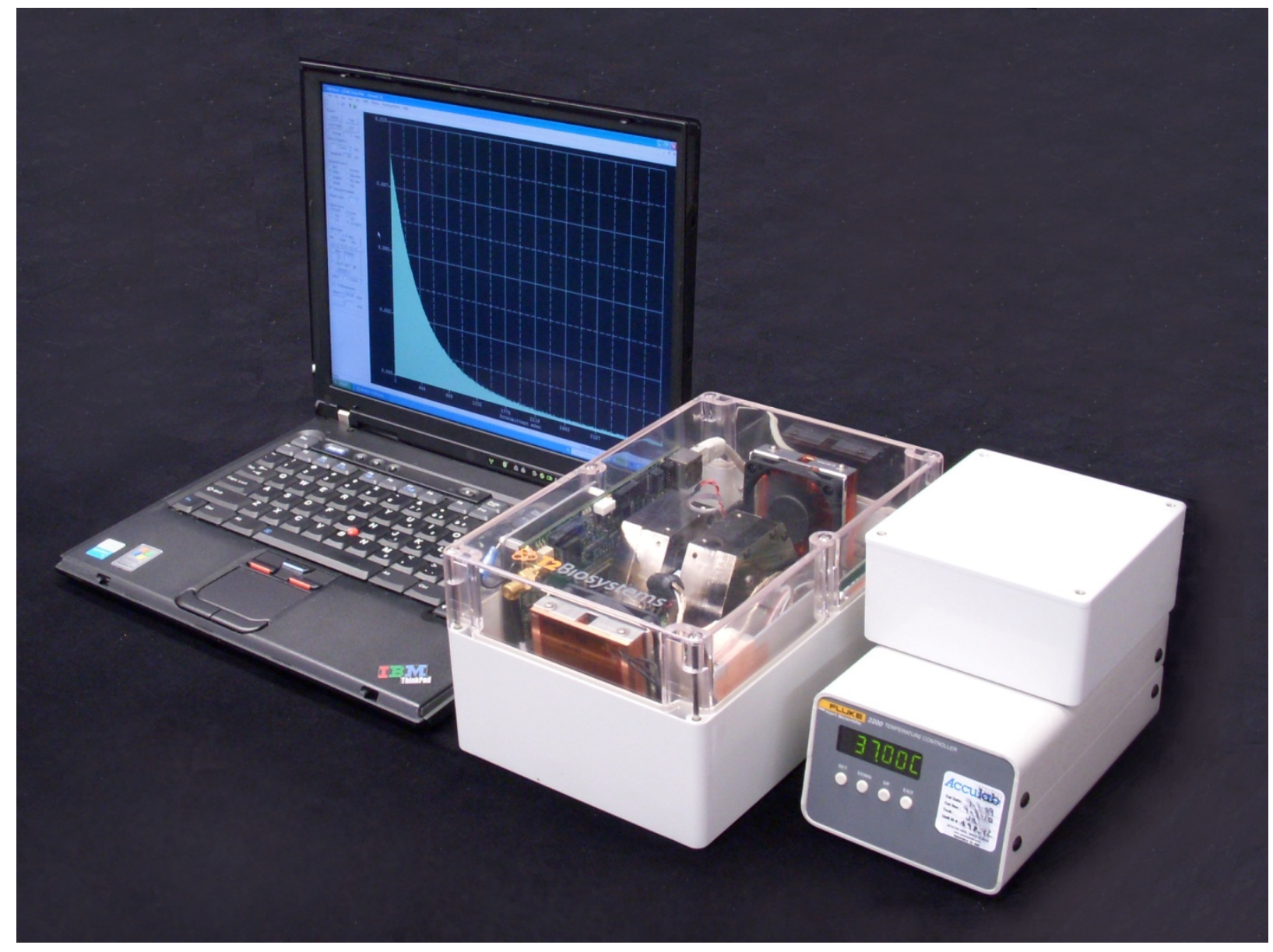

Figure 5. T2Bio's first temperature controlled T2 Reader ( $T_{2}$ relaxometer).

During the Automated and High Throughput builds, the need for stand-alone instrumentation to aid in flexible, manual assay development had grown and rather than being limited by the above-described 
Final Scientific \& Technical Report: Development of a System for Rapid Detection of Contaminants in Water Supplies Using Magnetic Resonance and Nanoparticles

shortcomings of the first T2 Reader design, an effort to improve the MR Reader was undertaken upon completion of those systems. The key goals of the redesign were to achieve the following:

1. Improve the system layout to gain better control of the MR signal pathway

2. Improve the temperature control

3. Remove costly, underperforming, and difficult to manufacture components

4. Integrate the system into single package

These goals were achieved by making several changes to the reader design. Most notably, the packaging and component layout were better arranged by means of using a custom enclosure. This led to a more optimal control of signal pathways and to a reduced system-to-system variation. Improvements were also made to the electronics. For example, the off-the-shelf preamplifiers were replaced to improve on their fair noise performance, simple protection circuit, and a sub-optimal signal pathway due to the preamplifiers being spread across separate boxes. Similarly, the temperature controller and power supply were moved from being in separate devices to being contained in the same enclosure. Several of these improved $\mathrm{T} 2$ Readers were built, shown to determine precise $\mathrm{T}_{2}$ relaxation times and thus used in assay development efforts.

\section{The Next Generation (NG) T2 Reader}

A block diagram of the components required for a MR measurement is shown in Figure 6 . All of these components except the RF transmitter and preamplifier were optimized prior to this project. The transmitter, which used an inductive coupling mechanism to the probe, was optimized by simplifying its cabling and probe circuitry to retain its excellent performance. The original preamplifier, which had fair noise performance, consisted of discrete off-the-shelf (OTS) modules and protection circuitry that was large, had higher power consumption than desired, and was sub-optimal for measuring small sample volumes. Another project at T2 Biosystems developed a low cost, low power, high performance preamplifier/transmit system for small sample measurements was developed to produce a single PCB incorporating both the preamplifier and transmitter. A new PCB, called RX/TX for receive/transmit, exhibited remarkable performance across a range of sample volumes and coil configurations. For example, it was used to measure samples of about $1 \mu \mathrm{l}$ in capillary tubes at signal-to-noise ratios in excess of 100, and also samples on a single-sided coil etched on a PCB. 
Final Scientific \& Technical Report: Development of a System for Rapid Detection of Contaminants in Water Supplies Using Magnetic Resonance and Nanoparticles

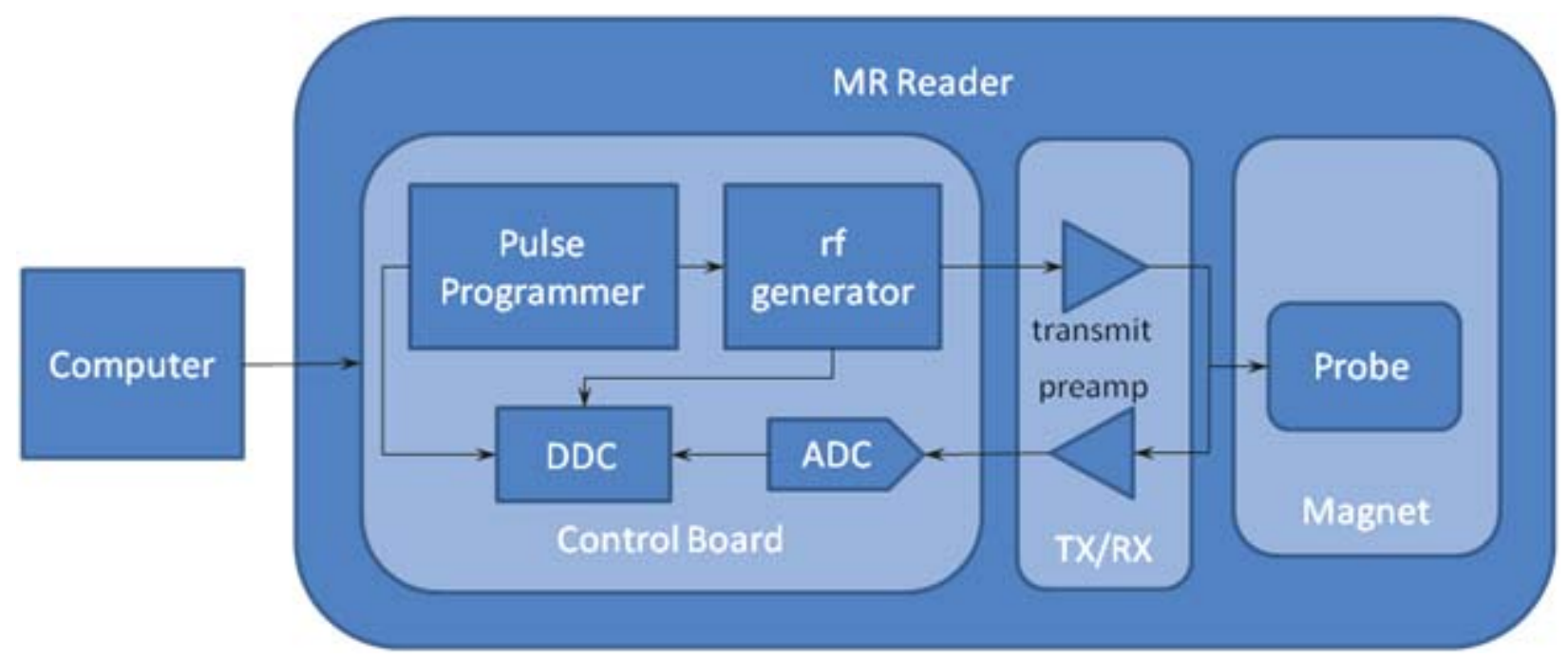

Figure 6. Components of a T2 Reader.

A comparison of the new RX/TX circuit and the original circuitry that performed the same functions is shown in Figure 7. The RX/TX circuit improves the signal-to-noise ratio (SNR) performance on small coils by about a factor of 5 , as well as improving packaging issues. Based on these improvements, this new RX/TX circuit was used in the design of a new generation T2 Reader.

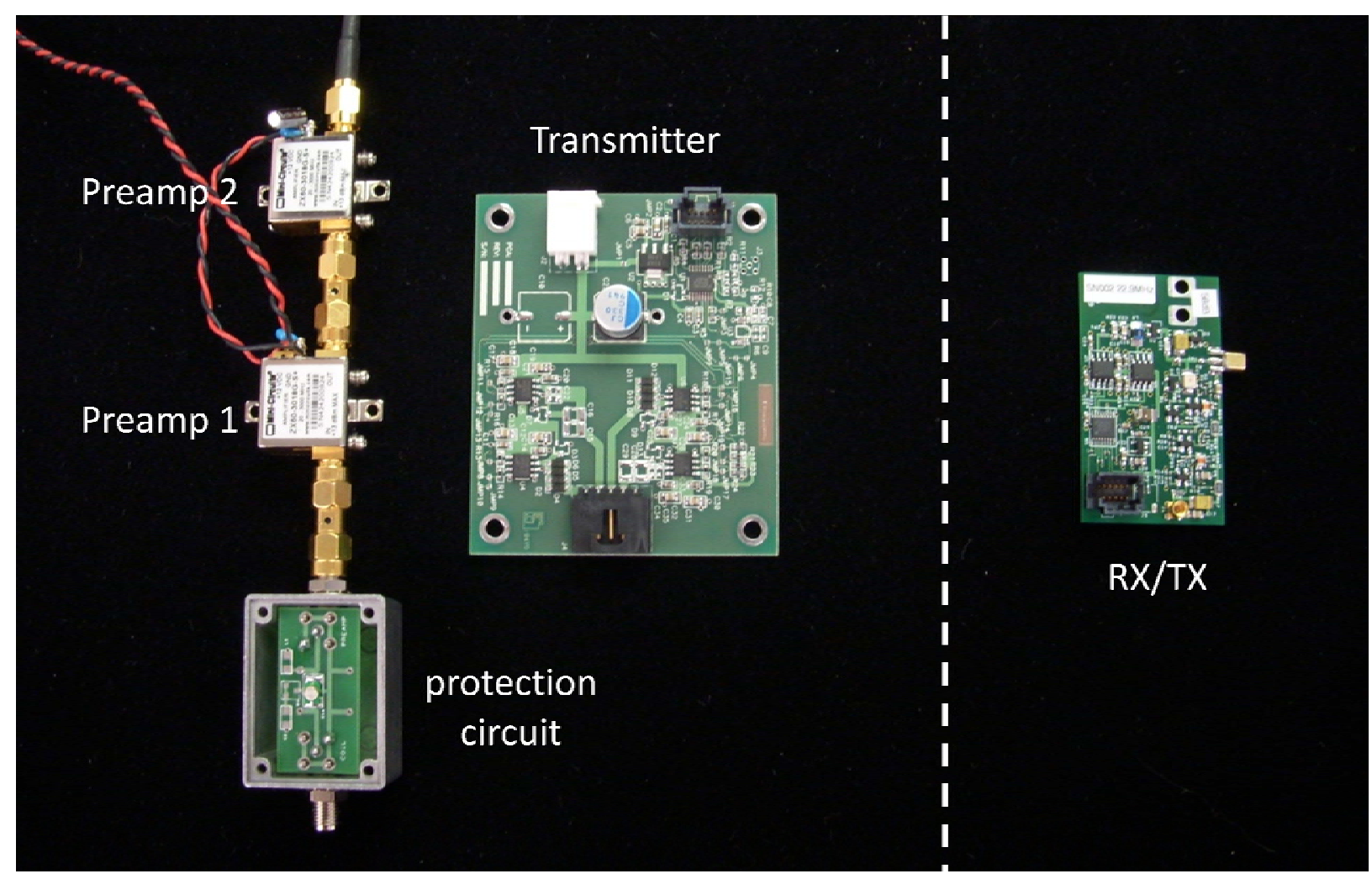

Figure 7. Original T2 Reader preamplifier chain and transmitter compared to the new RX/TX circuit that provides the same functionality. 
Final Scientific \& Technical Report: Development of a System for Rapid Detection of Contaminants in Water Supplies Using Magnetic Resonance and Nanoparticles

One noteworthy design feature of the new RX/TX circuit is its low power consumption. Three different design options were considered, as outlined in Table 1. The RX/TX circuit was designed to draw preamplifier power from the signal cable ( $50 \mathrm{Ohm}$ cable to the control board) and transmit power from the transmitter control cable (10 conductor ribbon cable also to the control board). While this arrangement was not a limitation for the preamplifier, the transmitter power, which had already been reduced by halving the number of stages, was further reduced by operating from a $5 \mathrm{~V}$ rather than a $12 \mathrm{~V}$ power rail.

Table 1. Possible system configurations with new RX/TX circuit.

\begin{tabular}{|l|l|l|}
\hline \multicolumn{1}{|c|}{ Configuration } & \multicolumn{1}{|c|}{ Pros } & \multicolumn{1}{c|}{ Cons } \\
\hline Same coil - transmitter on 5 V & $\begin{array}{l}\text { No hardware changes } \\
\text { Coil is typically under-filled }\end{array}$ & $\begin{array}{l}\text { Increased 5 V power draw } \\
\text { Longer pulse times (narrower bandwidth) } \\
\text { Longer preamp recovery } \\
\text { Shorter echo averaging time }\end{array}$ \\
\hline Same coil - transmitter on 12 V & $\begin{array}{l}\text { Unchanged coil } \\
\text { Nearly the same pulse times } \\
\text { power separate from acquisition } \\
\text { coil is typically under-filled }\end{array}$ & $\begin{array}{l}\text { Extra power supply and cabling } \\
\text { Possible added noise due power pathway }\end{array}$ \\
\hline Smaller coil - transmitter on 5 V & $\begin{array}{l}\text { Nearly the same pulse times } \\
\text { No extra cabling/signal pathways } \\
\text { Lower sample volume can be used }\end{array}$ & $\begin{array}{l}\text { Lower sample volume must be used? } \\
\text { Coil may be over-filled }\end{array}$ \\
\hline
\end{tabular}

The first configuration was given very little consideration because of its excessively narrow excitation bandwidth and corresponding shortened echo averaging time. The second configuration was believed to have lower signal integrity than the third configuration, because of the addition of another power supply. The third configuration was determined to be most favorable because the SNR for the RX/TX circuit was more than adequate for even very small ( $1 \mu \mathrm{l})$ samples, therefore it was not expected that sensitivity would be compromised using a smaller coil with this configuration.

Accordingly, a coil was designed to accommodate a $0.2 \mathrm{ml} \mathrm{PCR} \mathrm{tube} \mathrm{as} \mathrm{the} \mathrm{sample} \mathrm{holder.} \mathrm{The} \mathrm{coils}$ were sized and inserted into a mold such that only the bottom portion of the PCR tube would penetrate the coil. At $20 \mu$ the height of fluid in the PCR tube filled the coil (see Figure 8). To address whether 'over-filling' the coil, or having excess sample material outside extending beyond the region of uniform field in the coil, would lead to a change in signal-to-noise (SNR) and decrease in measured $\mathrm{T}_{2}$ values it was determined that the effect of over-filling on the measured $T_{2}$ values was insignificant ( $\left.1 \%\right)$ and that the SNR scaled linearly with volume below the match fill volume of $20 \mu$ l. A typical SNR for a $20 \mu l$ sample was $>900$. Larger samples typically had only slightly larger SNR values because the coil was nearly full at $20 \mu$ l. At volumes below $20 \mu$ the SNR scaled linearly with volume. 
Final Scientific \& Technical Report: Development of a System for Rapid Detection of Contaminants in Water Supplies Using Magnetic Resonance and Nanoparticles



Figure 8. $0.2 \mu \mathrm{I}$ PCR tube with MR Coil.

The RX/TX circuit was modified only slightly to allow direct mounting to the probe and magnet resulting in a very compact arrangement (Figure 9). This arrangement required only two simple cables running from the RX/TX board to the control board. Replacing the distributed transmitter and preamplifier elements with the integrated RX/TX provided much better control of the signal pathway and addressed cost, power, and SNR performance. 
Final Scientific \& Technical Report: Development of a System for Rapid Detection of Contaminants in Water Supplies Using Magnetic Resonance and Nanoparticles

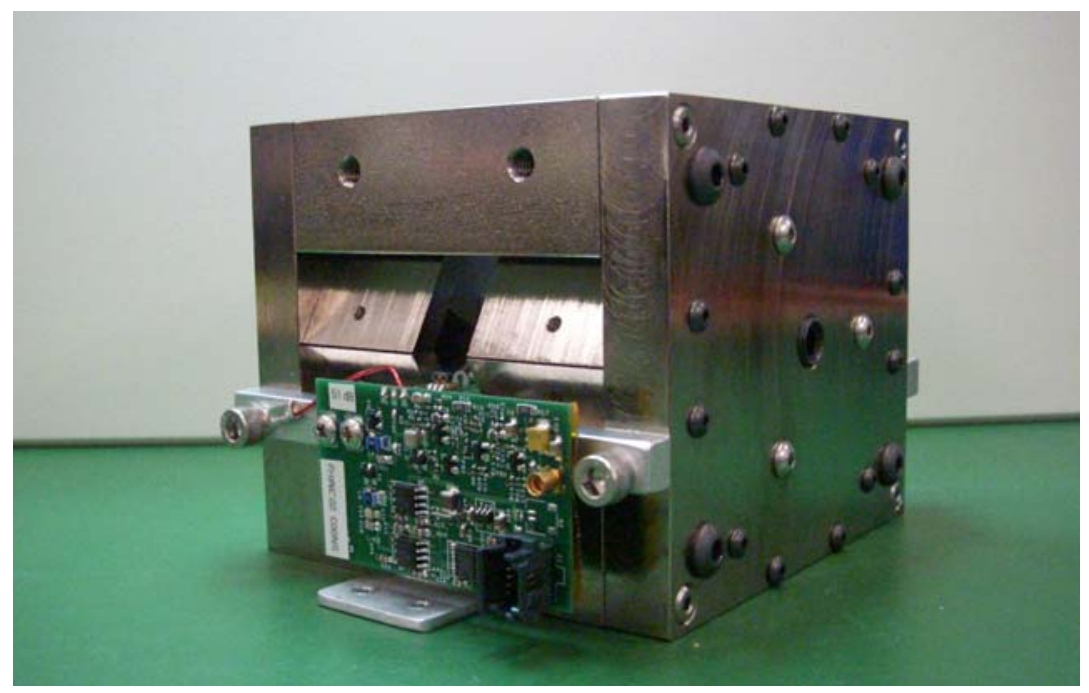

Figure 9. Magnet, probe, and RX/TX circuit.

The new T2 Reader design improved the temperature control of the assembly. The temperature control of the first $\mathrm{T} 2$ reader was largely limited by the packaging and air flow. Temperature management was improved by using a different system layout as well as larger heat sink elements and to improve both heat transfer and air flow. The system was designed to segregate the magnet assembly (magnet, probe, and RX/TX board) from the MR controller, the temperature controller, fans, and the power entry and conditioning. This served two purposes, first to isolate the magnet assembly from the electromagnetic interference (EMI) associated with the control elements and to provide a means of air circulation that would draw the waste heat produced by the controllers and power supply through the heater elements, to become part of the thermal path. The layout of this second generation T2 Reader (called NG Reader) is shown in Figure 10. Related changes to the temperature management system reduced the background electronic noise of the T2 Reader. The original T2 Reader was compromised by electromagnetic noise generated by the fans used to circulate air within the enclosure. The source of this electromagnetic noise was believed to be from the electromagnetic components of the fans, which were in close proximity to the MR magnet. Their rotation caused a very slight, but noticeable modulation in the MR static field when the fans were running. Although ferrous shielding was used to mitigate the problem in the original assembly, the new design used a different layout to place fan and heat fins, which acted as a heat sink, in a manner that was expected to provide improved air flow and reduce the electromagnetic noise generated by the fans. In addition, two hardware components were substituted to reduce the overall noise of the system: AC fans were used and an OTS pulse-widthmodulated controller that used a zero-crossing, AC solid state relay to drive the heaters. The zerocrossing feature of the relay minimzed switching transients by switching only when the voltage in the $A C$ power was at zero. 


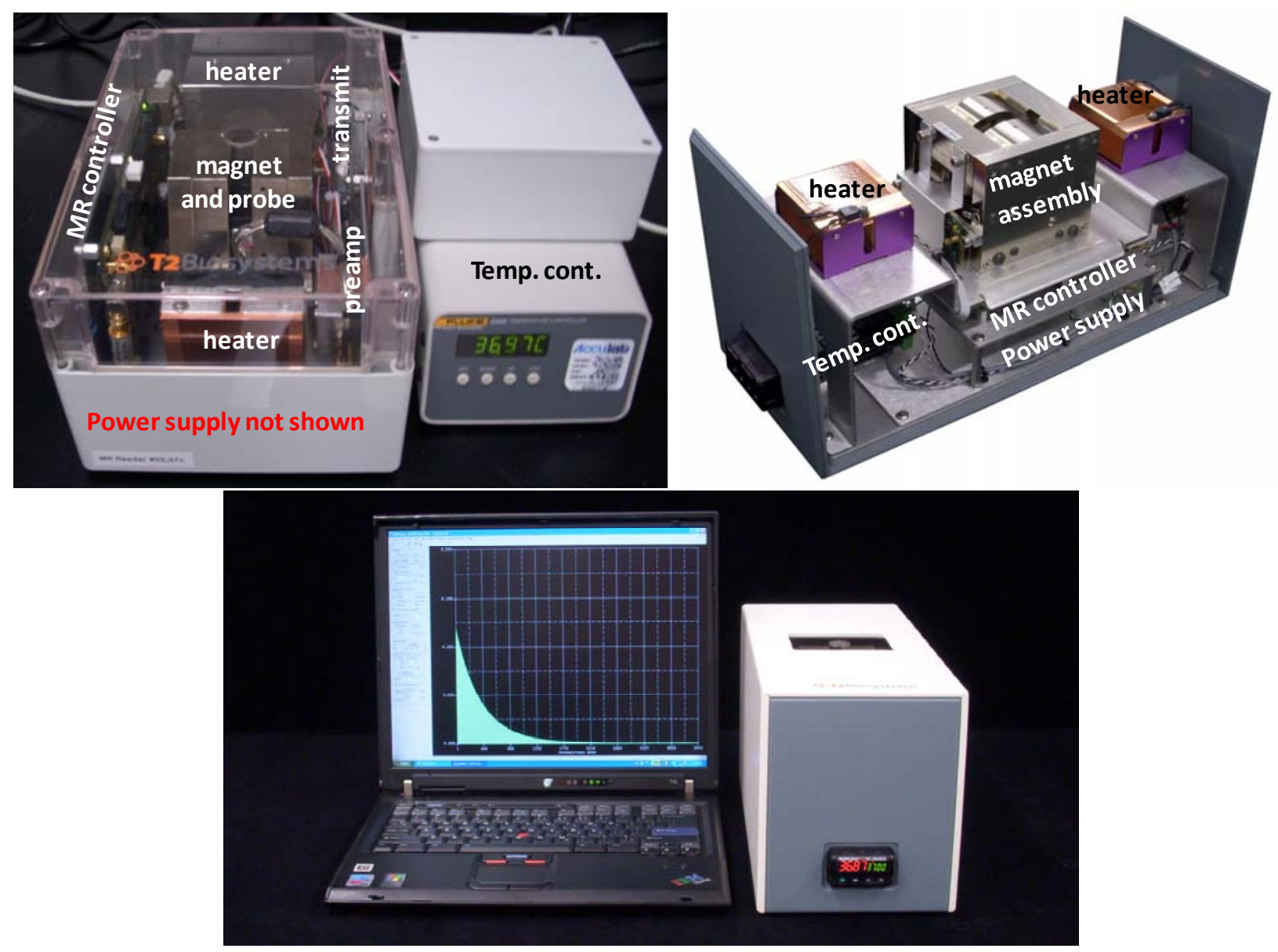

Figure 10. Original T2 Reader (top left) and NG Reader layout (Top right) and complete NG Reader system (bottom) after redesign under this project.

\section{Performance Testing of the "NG" T2 Reader}

One of the most critical performance parameters for a $T 2$ Reader is its $T_{2}$ measurement precision. Accordingly, the goal for T2 Reader precision was to maintain a level of precision that is 2-4 times higher than what can be achieved in other assay processes, such as fluidic handling. This was set to ensure that the $\mathrm{T}_{2}$ measurement contributes no more than $25 \%$ of the total error, if not far less. Accordingly, a metric of $0.5 \%$ coefficient of variation (CV) in $\mathrm{T}_{2}$ was chosen as a requirement for this system. Modeling showed and measurements confirmed that an SNR of approximately 130 was sufficient to achieve this level of precision using nominal pulse sequence and acquisition parameters. As stated above, the SNR for the new T2 Reader was much larger than 130 even for sample volumes as low as $5 \mu$ l.

The precision of the T2 Readers was measured by performing repeated measurements with a liquid standard, composed of a known, stable substance. This approach attempted to isolate T2 Reader performance from other variables that can affect the measured $T_{2}$ value, such as temperature fluctuations and biological sample instabilities. Exemplary results from this testing are shown in Figure 9, where an oil standard (light mineral oil - MOL) was used. This standard has a low evaporation rate 
Final Scientific \& Technical Report: Development of a System for Rapid Detection of Contaminants in Water Supplies Using Magnetic Resonance and Nanoparticles

and is fairly stable over time. Other oils with different viscosities were used to provide a range of measured $T_{2}$ values for complete characterization of the systems $T_{2}$ precision (data not shown).

The data from multiple runs were processed to yield measured $T_{2}$ values and other information such as noise level, SNR, and quality of the single exponential fit. The average $\mathrm{T}_{2}$ and the overall CV for the measurements summarized in Figure 11 were $199.5 \mathrm{~ms}$ and $0.06 \%$, respectively. The trend in the $\mathrm{T}_{2}$ values for this particular run show an even tighter CV over short timescales. It is possible that part of the total CV across the $4 \mathrm{hr}$ measurement period was due to small fluctuations in temperature inside the T2 Reader. The light mineral oil standard had a $\mathrm{T}_{2}$ temperature coefficient of about $2.5 \% /{ }^{\circ} \mathrm{C}$, so even a fluctuation of $<0.1^{\circ} \mathrm{C}$, which is consistent with the very tight temperature control of this design, could account for the excursions in the results over the $4 \mathrm{hr}$ measurement period. The CV of $0.06 \%$ of this run was better than the average of about $0.1-0.2 \%$ seen across many instruments and many runs. These $\mathrm{CV}$ values are remarkable for $\mathrm{T}_{2}$ relaxometers, and exceed the required $\mathrm{CV}$ of $0.5 \%$. Several $\mathrm{NG}$ Reader units were constructed and the measured variation in $\mathrm{T}_{2}$ precision was always better than $0.5 \%$ across all sample types and volumes measured.
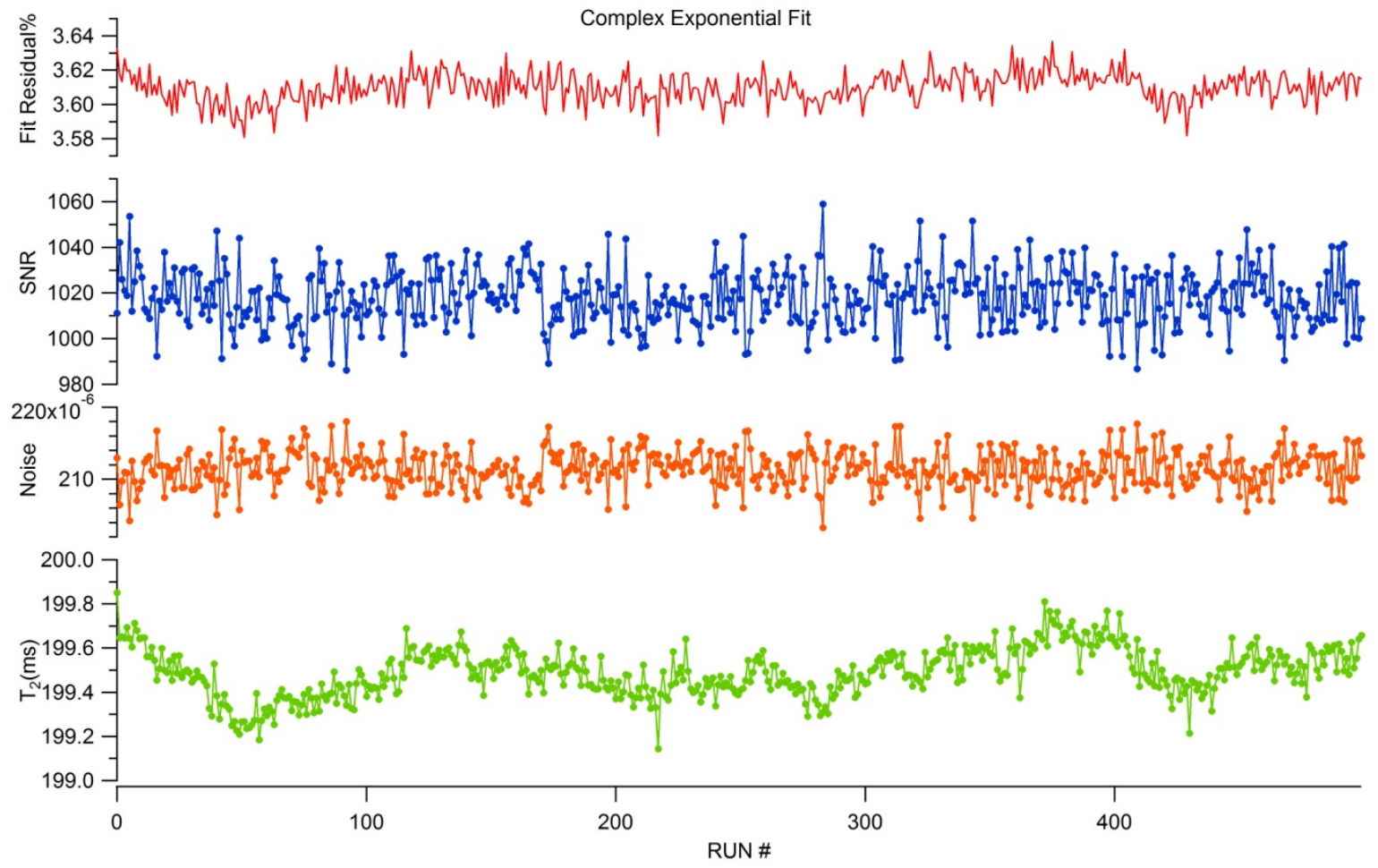

Figure 11. $\mathrm{A} \mathrm{T}_{2}$ precision measurement of a NG Reader (S.N. NG1003). The measurement was performed on a $20 \mu$ light mineral oil sample.

The accuracy across eight instruments was also measured. Three sample types at three different volumes were tested following the same method as used for Figure 11. The CV for each run was better than $0.5 \%$. The average $T_{2}$ value for each measurement set is reported in Table 2 according to sample type, sample volume, and instrument. Taking into account all eight instrument the CV's for any specific T2 Biosystems 
Final Scientific \& Technical Report: Development of a System for Rapid Detection of Contaminants in Water Supplies Using Magnetic Resonance and Nanoparticles

sample were never greater than $1.6 \%$. Because of the tight CV for each instrument, the variation in measured $\mathrm{T}_{2}$ values could easily be calibrated out by a standard calibrator across instruments.

Table 2. Instrument-to-instrument $T_{2}$ accuracy comparison for several NG Reader units.

\begin{tabular}{|c|c|c|c|c|c|c|c|c|c|}
\hline & \multicolumn{9}{|c|}{ T2 Summary Across Instruments } \\
\hline & MOH_10ul & MOL_10ul & MSO_10ul & MOH_2Oul & MOL_2Oul & MSO_2Oul & $\mathrm{MOH} \_40 \mathrm{ul}$ & MOL_40ul & MSO_40ul \\
\hline NG_1003 & 61.960638 & 198.57272 & 596.37076 & 62.086936 & 199.49136 & 600.29854 & \begin{tabular}{ll|}
61.39878 \\
\end{tabular} & 198.55776 & 591.2211 \\
\hline NG_1004 & 61.493864 & 195.86322 & 580.79622 & 61.094188 & 194.93206 & 586.52386 & 61.509656 & 196.62868 & 590.72454 \\
\hline NG_1005 & 63.423274 & 200.85184 & 597.93634 & 62.846894 & 198.99708 & 598.1372 & 62.626228 & 198.00022 & 584.51504 \\
\hline NG_1007 & 61.940116 & 197.16192 & 590.59854 & 61.737494 & 198.71192 & 595.10512 & & & \\
\hline NG_1008 & 60.965048 & 195.46556 & 590.23248 & 60.95678 & 196.70452 & 594.09072 & 61.365968 & 197.27284 & 594.58124 \\
\hline NG_1009 & 63.814175 & 202.9351 & 604.66224 & 63.567032 & 202.66108 & 603.9372 & 63.031752 & 201.41108 & 605.39268 \\
\hline NG_1010 & 62.932836 & 199.6834 & 595.42104 & 62.446272 & 198.94576 & 596.15256 & 61.170492 & 196.55972 & 586.37016 \\
\hline NG_1011 & 62.183616 & 198.72204 & 595.50836 & 61.7562 & 197.81424 & 596.8514 & 61.27236 & 197.6794 & 590.16192 \\
\hline Average T2 & 62.3391959 & 198.656975 & 593.940748 & 62.0614745 & 198.532253 & 596.387075 & 61.7678909 & 198.015671 & 591.852383 \\
\hline Stdev & 0.97342119 & 2.51878805 & 6.96079838 & 0.87720771 & 2.24257414 & 5.06863103 & 0.74173152 & 1.65988528 & 6.82160646 \\
\hline$\% \mathrm{CV}$ & $1.56 \%$ & $1.27 \%$ & $1.17 \%$ & $1.41 \%$ & $1.13 \%$ & $0.85 \%$ & $1.20 \%$ & $0.84 \%$ & $1.15 \%$ \\
\hline & & & & & & & & & \\
\hline & & & & & Average & CV Across & nts on ALI & L runs & $1.06 \%$ \\
\hline & & & & & & & & & \\
\hline Across ALL v & & & & & & & & & \\
\hline Average T2 & 62.0687217 & 198.418414 & 594.156055 & & & & & & \\
\hline Stdev & 0.86706018 & 2.10885271 & 6.30854697 & & & & & & \\
\hline$\% \mathrm{CV}$ & $1.40 \%$ & $1.06 \%$ & $1.06 \%$ & & & & & & \\
\hline
\end{tabular}

\section{NG T2 Reader Summary}

In addition to the work performed to optimize the T2 Reader described above, the NG T2 reader was employed in the assay development described below. These tests, and others, demonstrate that the T2 Reader can provide superior reproducibility, precision, and ease of use over the existing non-portable commercial $\mathrm{T}_{2}$ relaxometer. The NG T2 Reader was a substantial improvement over the first integrated MR Reader built by T2Bio. The system had much better performance, in terms of $\mathrm{T}_{2}$ precision and temperature stability, and was much easier to build than its predecessor. The cost of goods was also decreased to be a fraction of the cost of commercial counterparts even in very low production quantities.

\subsection{Automated Bench-top Unit}

Having built and confirmed the efficacy of four of the first generation T2 Readers (Figure 5) as research tools, future instrumentation needs were considered. Two immediate needs were identified, the first was a fully automated system that could validate assays beyond laboratory research, and the second was a high throughput system that would perform a number of repetitive and time consuming tasks required during assay development thus increasing the productivity of development staff. Both projects were initiated before this program, but much of the work, including design and modifications, were completed as part of this program to help meet its objectives. The automated bench-top unit is discussed in this section, the high throughput system is discussed in the following section.

The automated bench-top system integrates the T2 Reader with all of the fluidic processing steps to fully automate all assay processing steps. Because the system was intended to serve as a development platform, one of the key requirements was versatility without significant hardware modification. Accordingly, three design requirements were set: (1) the cartridge itself had to be versatile; (2) the 
Final Scientific \& Technical Report: Development of a System for Rapid Detection of Contaminants in Water Supplies Using Magnetic Resonance and Nanoparticles

control software had to be easy to modify to meet new protocols as they were developed; and (3) the system had to contain the tools necessary to perform all basic fluidic and sample manipulations.

The first design requirement was met by using an 'open' cartridge architecture (Figure 12). The open cartridge consisted of a grid of removable reagent and reaction wells. The reagents (including sample) would be loaded by the user and sealed with foil prior to the run. The reaction wells were empty when loaded and had custom, slit-top caps that allowed reagent dispensing (and aspiration if required). The cartridge was constructed in such a way that a gripper mechanism could access any of the reaction wells and move them throughout the system for processing.

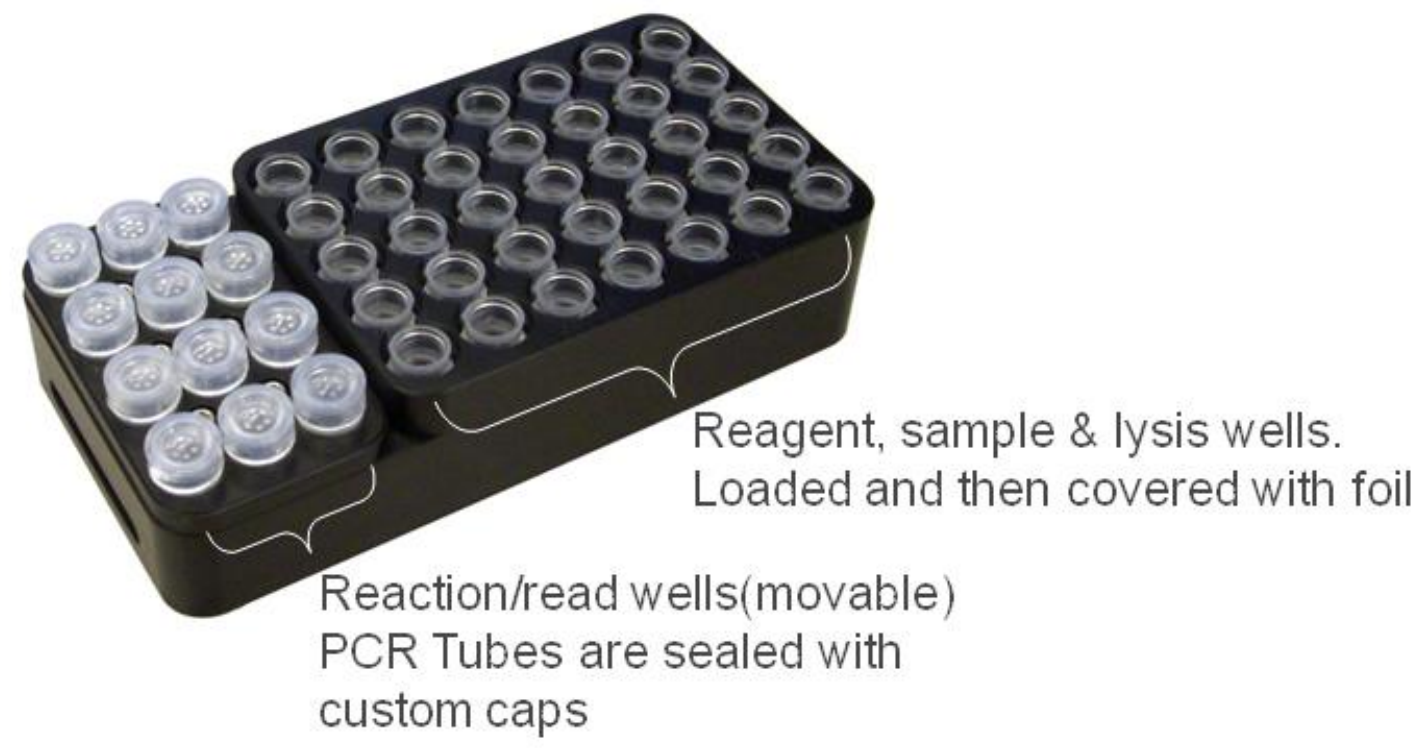

Figure 12. The Automated system cartridge.

The second design requirement was met by designing the system software so the user could completely specify system actions using a series of simple commands such as move, aspirate, dispense, etc., each with parameters detailing a fine level of control. This degree of granularity enabled a user to completely define an assay protocol, by assembling a simple text file in a process that could be completed in about an hour. The following text is an example of transferring a reagent to a sample well and includes the pick-up and removal of a pipette tip. The parameters following the aspirate and dispense commands specify volume, rate, starting height, etc.

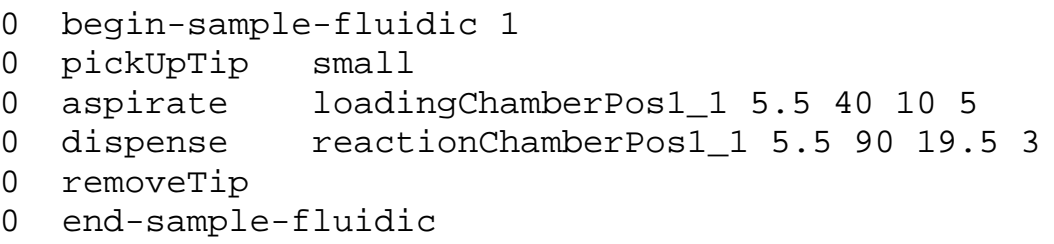

Another critical element of the software design was scheduling. Many processes such as incubation and mixing were time critical. The software tracked usage on all system stations including the manipulator T2 Biosystems 
Final Scientific \& Technical Report: Development of a System for Rapid Detection of Contaminants in Water Supplies Using Magnetic Resonance and Nanoparticles

(robot) to ensure timing was appropriate and maintained. Two software elements were developed to meet this need. The first element was a component of the automated system control software that scheduled the actions of the automated system. The second software element was a standalone application for modeling system throughput and station utilization which tested validity of a protocol and allowed instrument designers to 'size' stations to achieve a specific throughput.

\section{Automated System Hardware}

A block diagram and layout of the automated system hardware is shown in Figure 13. The system contained a superset of possible sample manipulations stations, allowing for significant flexibility in assay workflow design and execution during the prototype stage of assay and instrument development. The system was designed to be housed in a temperature controlled enclosure to provide isolation from the environment for temperature critical processes, namely fluidic transfer and $T_{2}$ measurements. $A$ three axis robot supporting fluidic handling (pipetter) and a reaction well gripper accessed all the system components. The fluidic manipulation station was designed to handle $5 \mu \mathrm{l}$ to $125 \mu$ l volumes with a precision of $1 \%$ or better using a commercial pipetter. A semi-custom temperature controller was fabricated to provide temperature control to six independent zones: 3 incubators (one with a mixing capability), a magnetic assisted agglomeration station, the MR Reader, and the enclosure. The system was intended for climate controlled environments so all temperature control was heat-only. The MR Reader used the same hardware components as the first MR Reader, but in a slightly different layout. Three incubation stations with independent temperature control could be configured according to the assay workflow. Three stations were included to allow for PCR thermocycling by moving the reagent wells between stations. Both gentle agitation (heated) and vortexing stations were included as well as two magnetic manipulation stations, one for magnetic assisted agglomeration and another for separation. Disposable materials including cartridges, pipette tips, and any wash buffers were transferred in an out of the system through a drawer. A disposal bin held used pipette tips and all fluids, with the exception of wash buffers, remained in the cartridge wells (albeit transferred from one well to another). Wash buffers used larger vessels for storage and disposal.
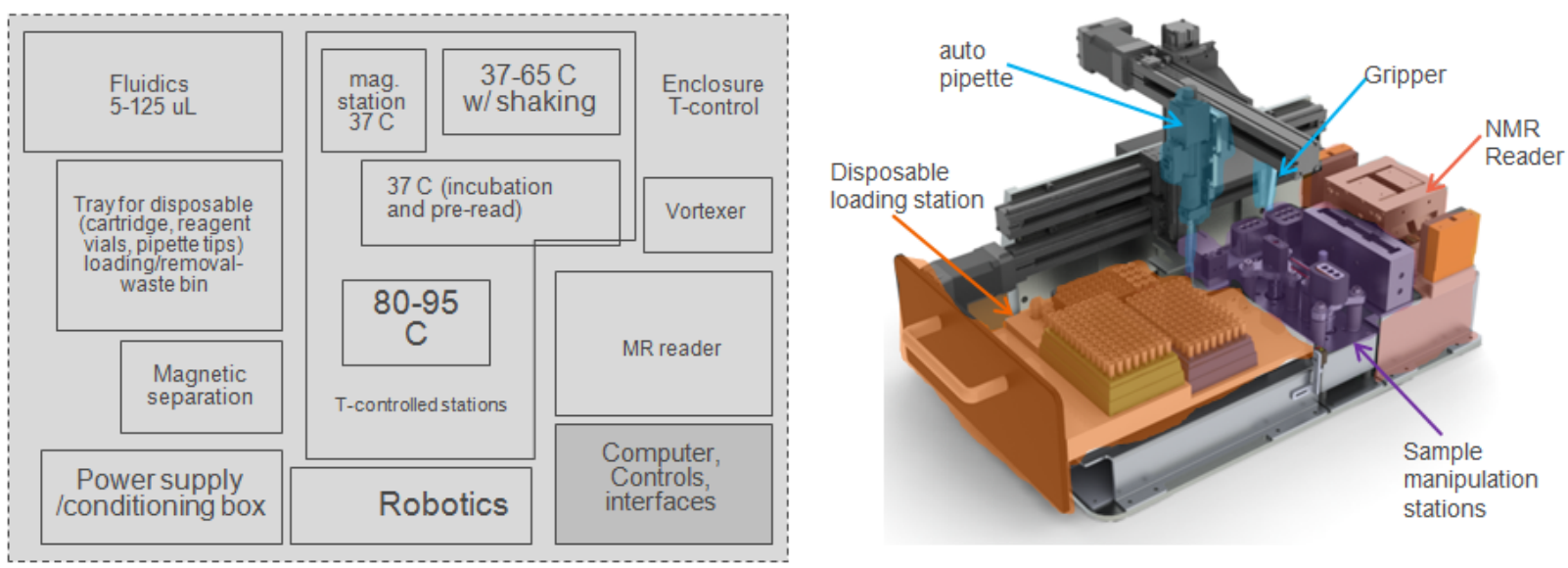

Figure 13. Automated system hardware. 
Final Scientific \& Technical Report: Development of a System for Rapid Detection of Contaminants in Water Supplies Using Magnetic Resonance and Nanoparticles

The completed system, shown in Figure 13, included a touchscreen monitor as the main user interface. It also had USB, external monitor, and Ethernet ports for alternate configurations. The system could also be controlled remotely using an Ethernet link.

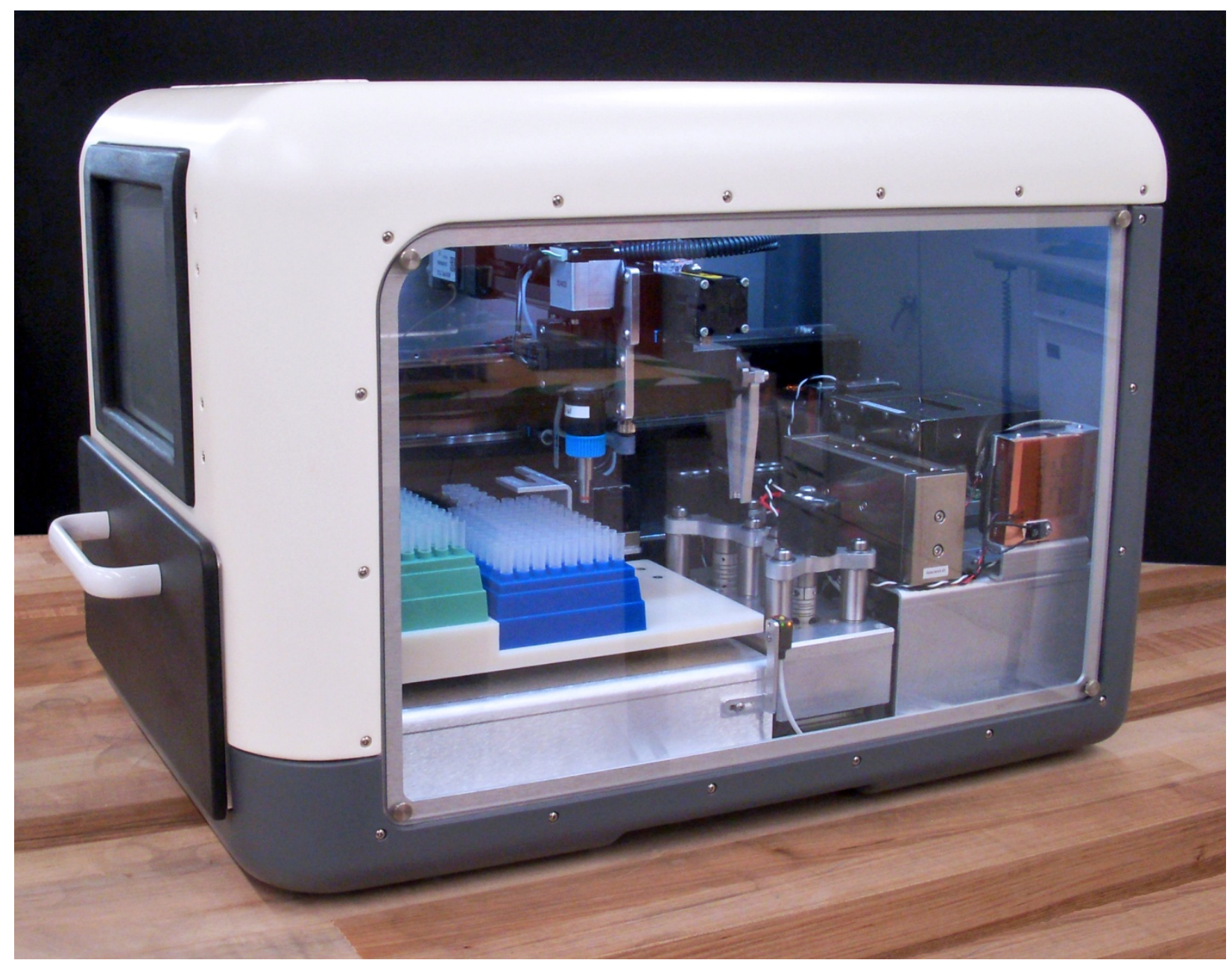

Figure 14. Integrated automated benchtop system.

\section{Automated System Summary}

A series of tests were performed on the integrated system to verify subsystems such as temperature control, fluidic transfer, $T_{2}$ measurement, and efficacy of processing stations (e.g., magnetic separation and agglomeration). System verification began with subsystem specific tests, such as gravimetric confirmation of fluid transfer and $\mathrm{T}_{2}$ measurements of a standard, and moved on to broader system tests, such as $T_{2}$ measurements of fluids containing nano-particles that were prepared using system fluidics, culminating in complete assay testing.

A model immunoassay was used for full system verification. The result was an overall instrument $\mathrm{T}_{2}$ precision of $0.9-2.5 \% \mathrm{CV}$. The pipetting error was found to be less than $1 \%$. The results were found to be superior to manual methods using the MR Reader due to the much higher degree of control of

\section{T2 Biosystems}


Final Scientific \& Technical Report: Development of a System for Rapid Detection of Contaminants in Water Supplies Using Magnetic Resonance and Nanoparticles

variables such as temperature and timing, and the improved precision of key stations such as fluidics. Testing of the automated system confirmed that automated fluidic handling was not only feasible but improved the overall assay performance.

Because of its low throughput, the system was inadequate for routine assay development. Rather than continuing efforts on an automated system, the majority of efforts were shifted to instrumentation that could serve as robust assay development platforms, the NG Reader and the High Throughput system. T2Bio continues to develop an automated instrument that will provide adequate throughput for assay development and field testing. The timeline for that development maps to this program's 3-year schedule.

\subsection{High Throughput Instrument}

Due to the large amount of sample processing required to fully verify and validate a diagnostic assay, achieving adequate throughput of tested assays is critical for a successful assay development program. Such high throughput is particularly critical for design of experiments and other multi-parameter studies. T2Bio set the objective of being able to easily process between 300-1000 measurements per day to allow for rapid assay development and optimization. Early in the development stage of an assay, prior to optimization, many of the sample processing steps and formulations require extensive testing to adequately explore the various parameters. Accordingly, it was decided to build an automated instrument that was capable of handling a high volume of post-fluidic assay processing steps, namely incubation, magnetic assisted agglomeration (MAA), mixing, and $T_{2}$ measurement. This would allow a number of researchers to separately adjust assay formulations at the bench and transfer the preparations to the High Throughput (HTP) instrument for final processing, freeing them from routine, time-consuming tasks. The design was conceived in advance of the initiation of this program, as the need for it became clear, engineering, testing, and modifications of the system were completed to enable the assay development completed as part of this project.

\section{HTP Sample Handling Hardware}

The initial HTP design consisted of incubation, mixing/vortexing, and $T_{2}$ measurement capability to support a throughput rate of more than 100 samples per hour. To process this many samples either multiple robots or a very high speed robot was required. To meet this need, T2Bio contracted with an outside firm (FTM Design) that had considerable experience implementing solutions with Epson's highspeed, pick-and-place, G-Series Scara robots. These robots have 4 axes of movement $(\Theta 1, \Theta 2, \Theta 3$, and $Z$ ), enabling it to move linearly in $X, Y$, and $Z$ directions, and rotate with respect to the $Z$ axis. They are capable of speeds in excess of $5 \mathrm{~m} / \mathrm{s}$ with placement accuracies of $\pm 25 \mu \mathrm{m}$ and \pm 0.005 degrees. The range of motion has a shape much like a cardioid and can cover nearly a square meter of deck space for instrument placement. The HTP system, shown in Figure 15, was designed around this robot, centered on a 42 " square deck surrounded by processing equipment (e.g., incubators, mixer, MR Reader). The volume under the deck was used for power entry and control equipment (e.g., the robot controller, computer, air compressor, and relay bank). The entire system was housed in a cabinet. The instrument deck was visible behind polycarbonate panels with doors on one side. Access to the instrument deck was software controlled to prevent exposure to an active robot. The user interfaced with the system using an external monitor, mouse, and keyboard.

T2 Biosystems

DOE project DE-SC0001821

Page 19 of 38 


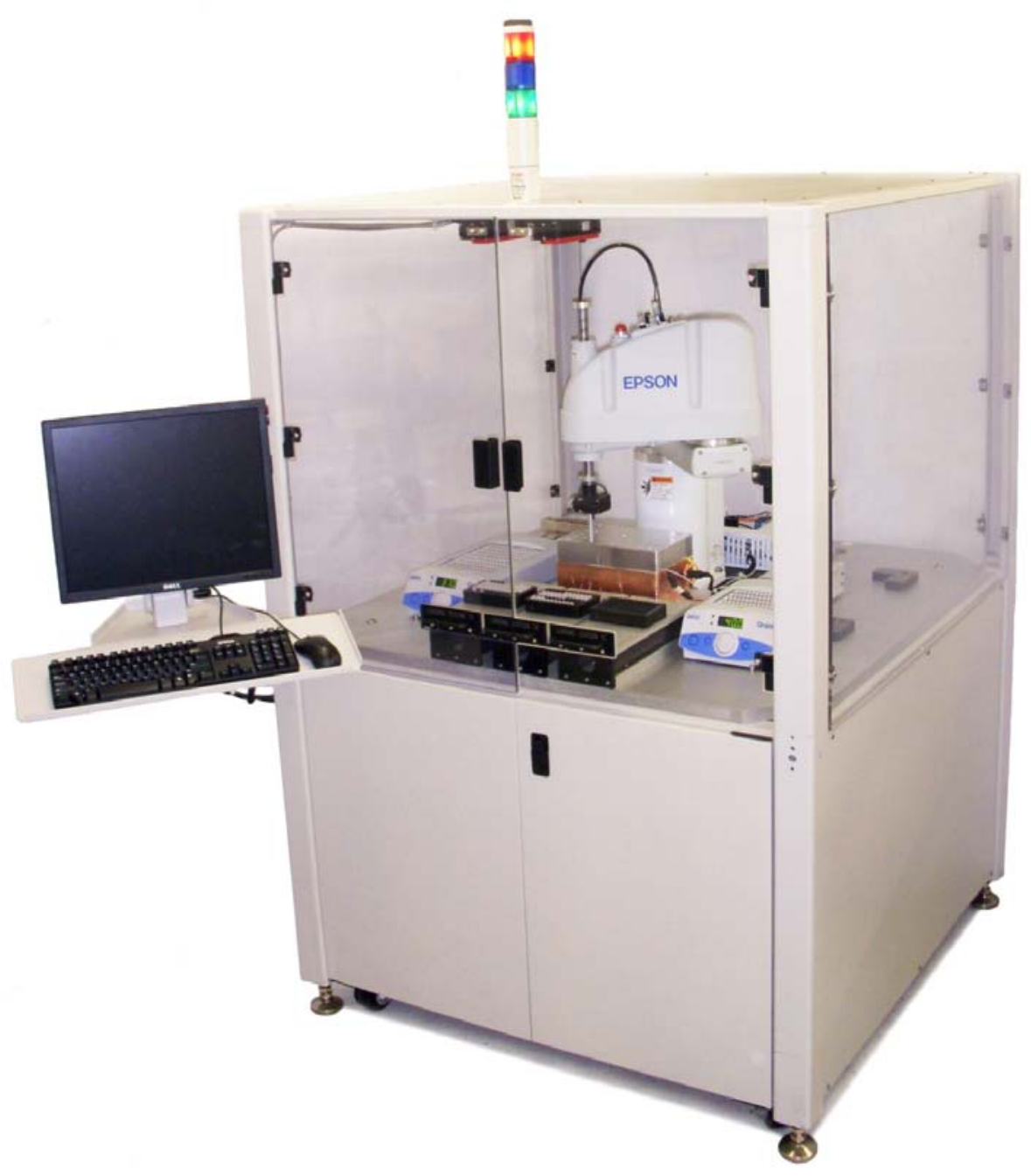

Figure 15. High Throughput system

In many cases the number of samples per experiment were much lower than 100 , so to fully utilize the possible throughput, the system had to serve multiple users at the same time. To this end, the system was designed to accommodate 3 separate loading trays, each loading through an independent drawer. The system controlled drawer access through its scheduling software. Samples were loaded into these drawers on a tray comprised of a compact 96-tube plate arrangement. The pick-and-place robot would then access and move tubes at specific positions for processing.

The original design called for multiple MR Readers to achieve the target throughput because a single $T_{2}$ measurement on a $150 \mu \mathrm{l}$ sample was expected to take $30-60 \mathrm{~s}$ to achieve. However, the improvements in the T2 Reader meant that a single $8 \mathrm{~s}$ scan would provide adequate SNR and precision, therefore, a single MR Reader could make >100 measurements per hour.

Off-the-shelf, Grant dry block heaters were selected for the incubators. Their blocks were modified to fit and register PCR tubes. Initially three incubators were installed, one 224 position, and two 112 
Final Scientific \& Technical Report: Development of a System for Rapid Detection of Contaminants in Water Supplies Using Magnetic Resonance and Nanoparticles

position models. Their temperature settings were user adjustable, but not tracked or controlled by the HTP system software.

A specialized mixer/vortexer was designed to meet the design requirement that it could start and stop at a specific location so that the robot could reproducibly pick and place samples. Off-the-shelf solutions were sought, none were found, so a custom module was designed. The orbital motion required for vortexing was somewhat mechanically complex and the first design that was implemented was both challenging to machine and assemble. An improved vortexer design (shown in Figure 16) reduced the machine tolerance on the parts and was much easier to assemble. Under use with typical duty cycles no failures or wear have been observed. The unit was installed in the HTP system in June of 2010 and has been operating without incident until the date of writing this report.

A vacuum gripper was selected for sample tube manipulation because the Epson Scara pick-and-place robot is often configured with this capability. Accordingly, an air

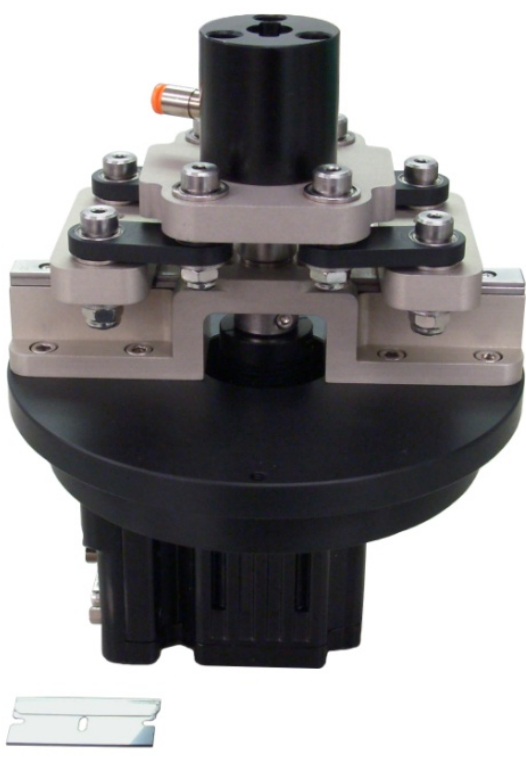

Figure 16. HTP Vortexer. compressor and vacuum valves were added to the system.

General purpose I/O lines in the robot controller were used for the valve interface.

\section{HTP System Software}

The control software for the system was comparable to the automated benchtop system described above, to the extent that much of the same software code was used. Similar to the automated system, the HTP required smart scheduling and adaptive workflows. In some respects the HTP system was simpler than the automated system because the operations are typically less complex, centering on movements and triggers (e.g., triggering an MR read) rather than complex fluidic transfers that include many tracking parameters. Developing a workflow (or assay profile) in the HTP system became less complex, as shown by the HTP's profile editor dialog in Figure 17. The pane on the left side of the dialog lists the HTP's current hardware configuration (list of components on the HTP deck). In this example, there are seven stations, three for magnetic assisted agglomeration (in various magnetic field geometries), two incubators, one T2 Reader, and one vortexer. The number of available positions in each station is listed (e.g., 112 positions in incubator 1). The pane on the right is a user designed workflow. It lists, in order, each station and duration used. Once written, the profiles can be saved and recalled when ready to run. 
Final Scientific \& Technical Report: Development of a System for Rapid Detection of Contaminants in Water Supplies Using Magnetic Resonance and Nanoparticles

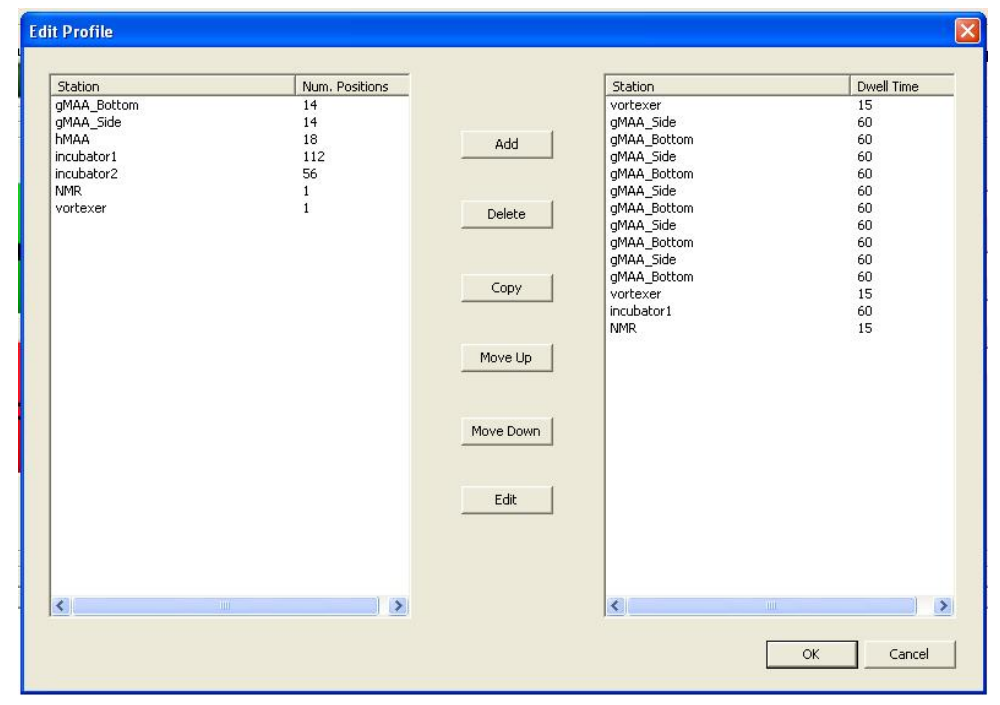

Figure 17. HTP system software's profile editor dialog.

The main window of the HTP system control software is shown in Figure 18. As depicted, the system was set up to run a profile called workflow3. A series of indicators across the top of the window displayed system status. A progress pane listed the duration of the profile and the elapsed and remaining times, and the profile being executed was displayed. In the pane on the right, labeled "experiment", the user entered specific details on the measurement set to be performed, such as number of samples and the tray(s) in which they were loaded

As shown in Figure 18, the experimental parameters for this specific experiment were: 24 loaded samples, 14 scheduled operations (e.g., vortexing, magnetic assisted agglomeration, incubation and $\mathrm{T}_{2}$ measurement), a single sample processing time of 705 seconds and a total experiment time of 79.18 minutes. 
Final Scientific \& Technical Report: Development of a System for Rapid Detection of Contaminants in Water Supplies Using Magnetic Resonance and Nanoparticles

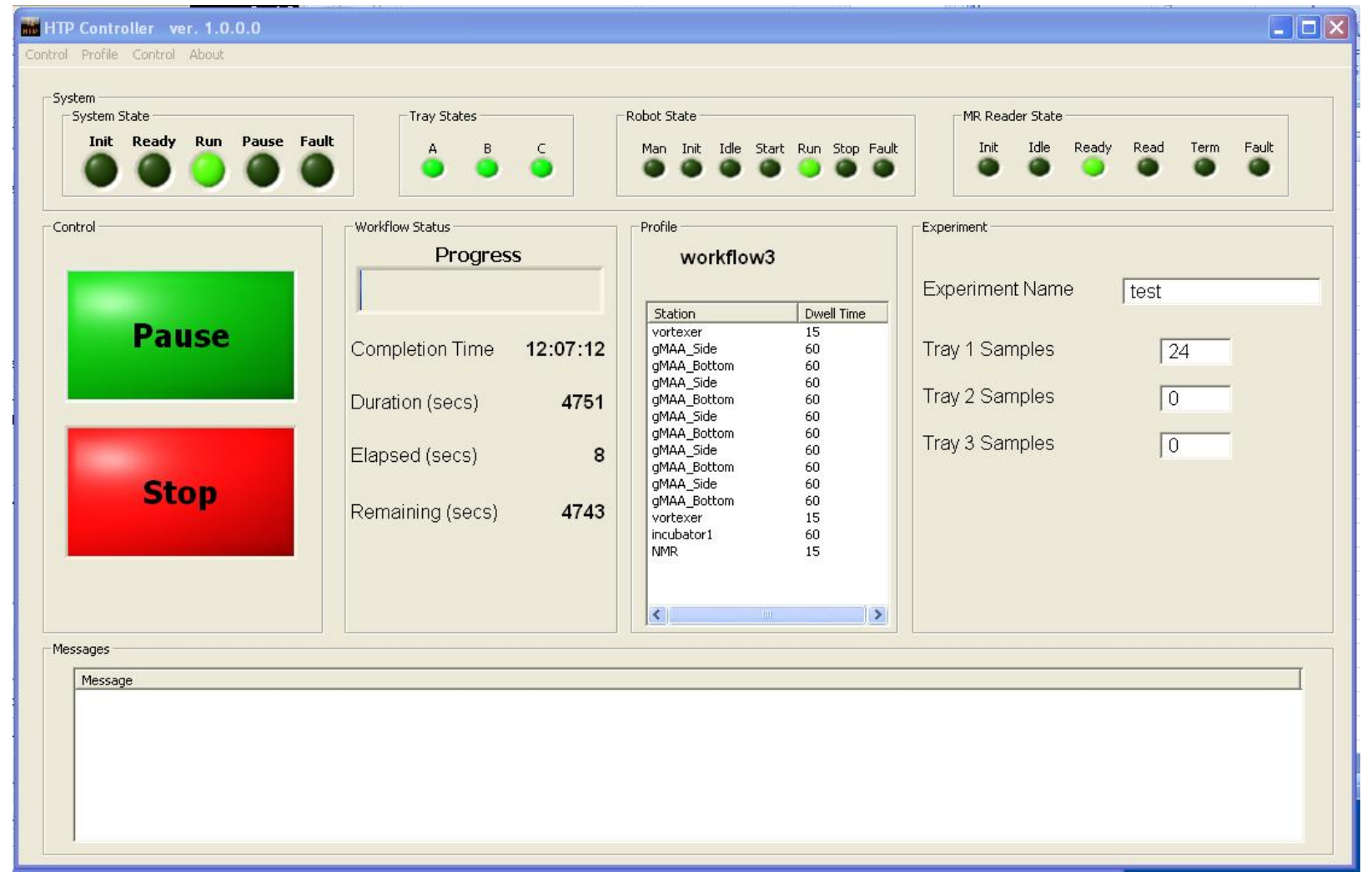

Figure 18. Main window of the HTP control software.

Although HTP was designed to be very simple for a user to run, its underlying scheduling software was intricate. The primary complexity was that the software had to find a combination of robotic steps that adhere to the timing requirements for each assay profile using the available resources on the deck. The scheduler determined the number of samples that could be stepped through a profile without violating timing. Knowing the sample flow in advance was found to be useful for designing the system and scoping out possible assay workflows. Therefore, a standalone version of the scheduler was also developed, which was called the Workflow Simulator (Figure 19).

The Workflow Simulator allowed users and designers to set a layout (station types and number of positions in each), specify a workflow and a number of samples to process, and then determine a schedule that adheres to the timing requirements of the assay workflow. The results of the simulator assisted in highlighting locations of potential bottlenecks formed in any assay workflow. In the example shown in Figure 19, the $20^{\text {th }}$ sample started its workflow about 1200 seconds after the first sample. The graphical display shows that the backlog in this workflow was the vortexer because it only had one position and requires a sample residence time of $15 \mathrm{~s}$. When the simulator was run again with 2 vortexers, the $20^{\text {th }}$ sample started its workflow 750 seconds after the first sample and the total duration was reduced from 2281 to 1516 seconds. Analyses like this were used to size the various workstations for optimal system utilization.

\section{T2 Biosystems}


Final Scientific \& Technical Report: Development of a System for Rapid Detection of Contaminants in Water Supplies Using Magnetic Resonance and Nanoparticles

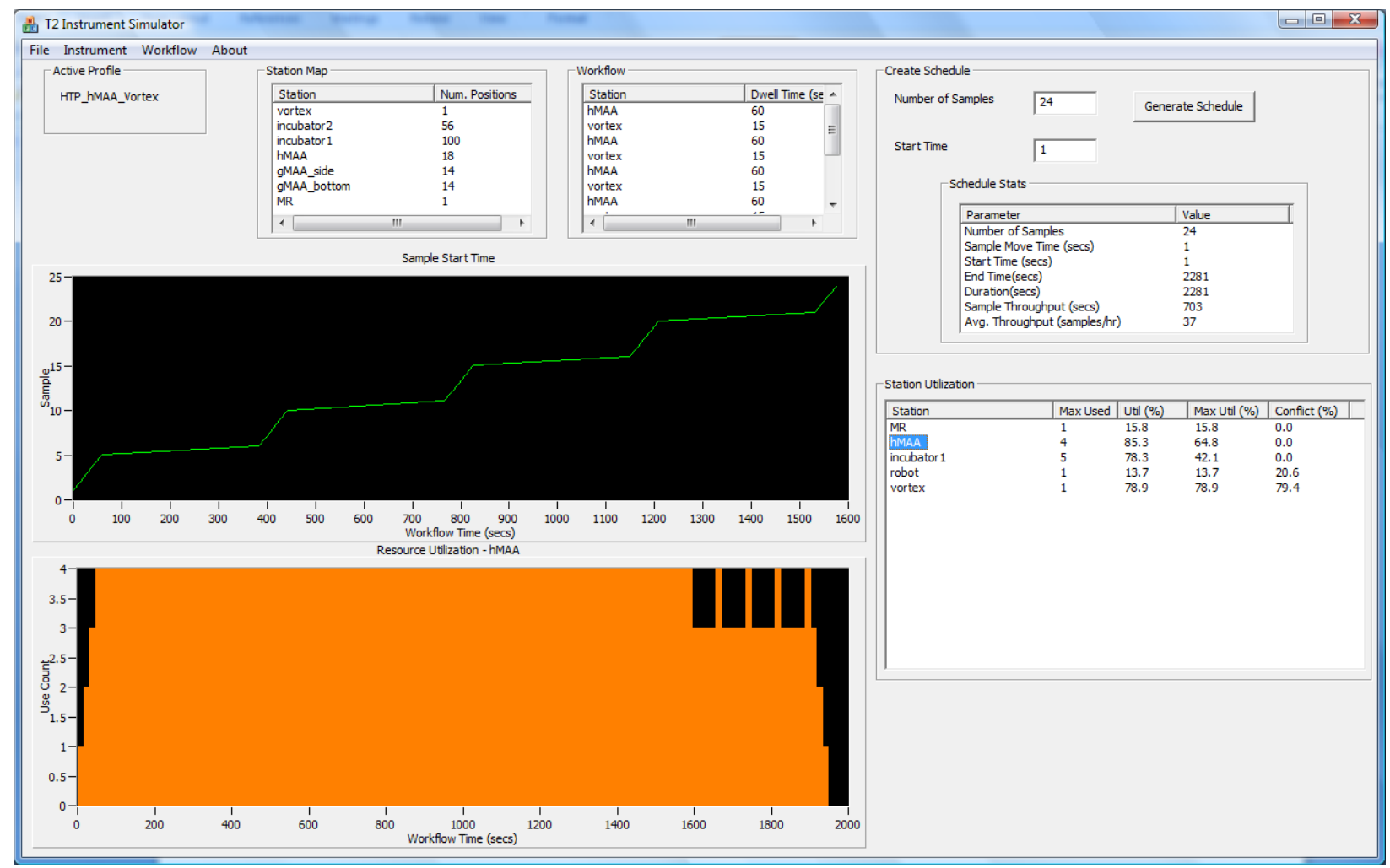

Figure 19. Workflow Simulator.

\section{HTP Reliability and Modifications}

The HTP system was constructed as a one-off breadboard and as such it had a few reliability issues as it was put into use. Three significant problems surfaced and were addressed before the system could be dependably used. They were as follows:

1. Undersized air compressor - the air compressor that was originally installed in the system could not produce the volume of air required to operate the vacuum valve. This was due to an error in the design calculation. The compressor was replaced and the problem was solved.

2. The system would fail intermittently - the cause was traced to a communication error (though log files) between the robot controller's CPU and the DSP servo controller. Ultimately the controller was replaced and the problem was solved.

3. Intermittent dropping of samples - much of this issue was due to slight inaccuracies in system mapping and no tolerance on the vacuum chuck alignment and two improvements were designed and employed. The first was to slightly modify the chuck (sub-millimeter) such that misalignment could be tolerated. The second was to develop an automated mapping routine that eliminated much of the human error involved in mapping a system with hundreds of positions. A modification to the vacuum chuck simply replaced a rigid aluminum contact with a flexible silicone bellows. While a flexible contact was actually specified in the original system design, it was deemed too large to be used in certain processing stations and it was replaced with the rigid chuck. As a result of installation of the flexible bellows and improvement of the station mapping dramatically decreased, but did not completely eliminate sample tube drops. 
Final Scientific \& Technical Report: Development of a System for Rapid Detection of Contaminants in Water Supplies Using Magnetic Resonance and Nanoparticles

The remaining problems are isolated to specific station positions and are quite likely due to tolerance stack-up in the tubes and the positions themselves. The occurrence is rare enough that is does not hamper the utility of the instrument.

All of these limitations were adequately addressed and the system has since then run robustly.

The HTP system was customized to allow for processing of molecular diagnostic (MolDx) assays by adding a station for homogeneous magnetic assisted agglomeration (hMAA). The Workflow Simulator, described above, was used to 'size' the station. Figure 20 shows a screenshot of the Workflow Simulator with a typical MoIDx workflow, hMAA for $600 \mathrm{~s}$, vortex for $15 \mathrm{~s}$, incubate for $60 \mathrm{~s}$, and $\mathrm{T}_{2}$ measurement. To achieve at least 300 samples per day, 10 hMAA positions were required. To achieve 1000, approximately 25 were required. The simulator shows that with 18 hMAA positions, approximately 700 samples could be processed in a single eight hour day. It was determined that this was a good compromise between throughput and deck space.

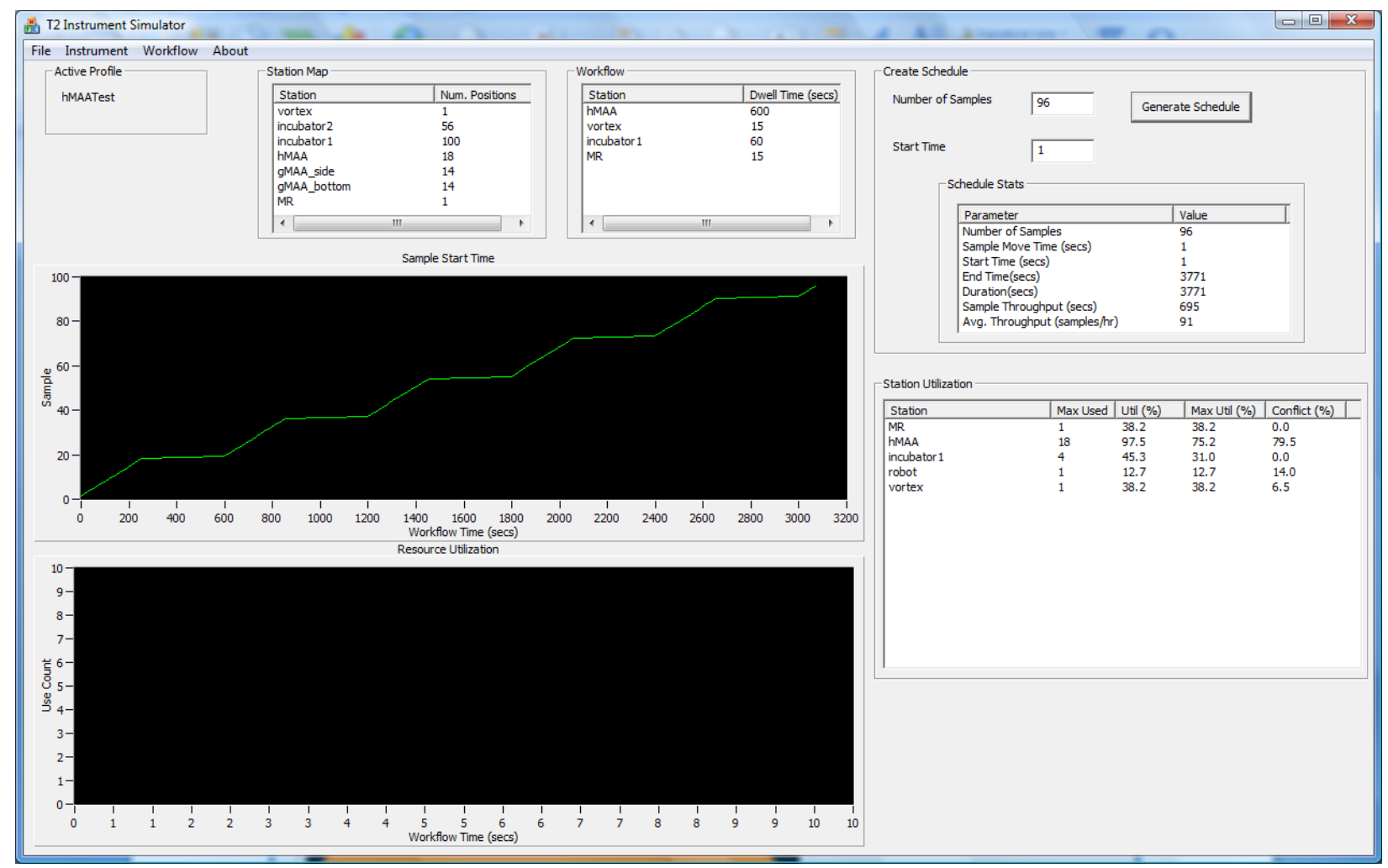

Figure 20. Workflow Simulator with MoIDX hMAA workflow sized for $>500$ samples per day.

Temperature control of the hMAA was implemented using an OTS controller and the same heater elements used in the NG T2 Readers. A polycarbonate housing was selected and designed so the user could easily inspect the stations during operation. The finished HTP-MAA unit is shown in Figure 21. The three units that make up the hMAA positions ( 6 each) are at the top. The middle section is the heat 
Final Scientific \& Technical Report: Development of a System for Rapid Detection of Contaminants in Water Supplies Using Magnetic Resonance and Nanoparticles

unit and the bottom is the 14 bottom and 14 side gMAA positions, which were components for a different project.

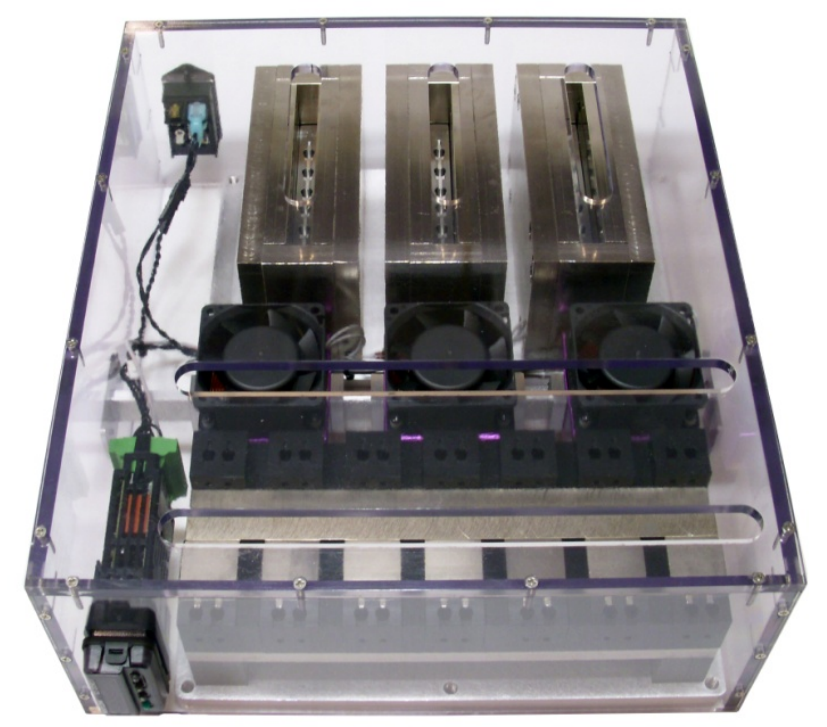

Figure 21. The HTP-MAA unit.

The final modification to the HTP system was the straight forward insertion of one of the NG Readers. It was added because of it improved performance. The original T2 Reader was not removed because there was enough deck space to accommodate both units.

\section{HTP Summary}

The HTP was T2Bio's answer to enable large volume testing early in the assay development cycle. The unit implemented common, time consuming tasks so the development scientist could spend more of their time on the upfront architecture. T2Bio developed the tools necessary for a user to modify a workflow in minutes. Additional tools were devised so that the system itself could be easily modified as the assay requirements evolved. The addition of MAA and the new NG T2 Reader were two such modifications. The performance of the system has improved as bugs are found and removed and they system has been continually available for the past several months. Depending on workflow, the HTP can process between several hundred to over 1000 samples in a day.

\subsection{Bacillus Thuringeinsis Assay Development}

The instrumentation described above was developed to enable the detection of nucleic acid targets in unprocessed water samples. As discussed above, a key advantage of the T2 Biosystems technology is the lack of extensive sample processing prior to detection. For nucleic acid tests, this manifests itself specifically in the lack of nucleic acid purification steps prior to amplification and detection. Nucleic acid purification procedures typically lead to a loss in target as manifest by a percent yield of the purification 
Final Scientific \& Technical Report: Development of a System for Rapid Detection of Contaminants in Water Supplies Using Magnetic Resonance and Nanoparticles

process that is significantly lower than $100 \%$. Target loss results in an increase in the overall limit of detection, which can lead to the requirement of larger sample volumes for processing in order to provide enough target for detection. T2Bio has demonstrated that nucleic acid based targets, such as bacteria or fungi, can be measured without nucleic acid purification by means of dirty-sample compatible amplification and detection methods.

In order to apply the T2Bio nucleic acid detection procedure to the analysis of nucleic acid targets in unprocessed water samples, Bacillus thuringeinsis (Bt) was selected as a model organism and Charles River Water (Cambridge, MA) was selected as the sample matrix. Bt is a member of the Bacillus cereus group. $B$. thuringeinsis is a common model organism due to two species of the Bacillus cereus group being mammalian pathogens ( $B$. cereus and $B$. anthracis). Additionally, the members of the Bacillus cereus group are nearly genetically identical. In fact, the classification of the six Bacillus species ( $B$. anthracis, B. cereus, B. thuringiensis, B. weihenstephanensis, B. mycoides, and B. pseudomycoides) has been fraught with debate with many advocating for the classification of $B$. cereus sensu stricto, $B$. cereus sensu lato, B. anthracis, and B. thuringiensis into a single species (Vilas-Bôas 2007). Others have advocated that there is sufficient genetic discrimination to merit separation as distinct species. Irrespective of whether they are classified as a single species with multiple variants or three separate species, they are genetically highly similar (Erlendur Helgason 2000) with the majority of their sequence difference confined to multiple extrachromosomal elements. This similarity was taken into account when designing the amplification probes and hybridization primers for the assays.

The following sections detail the method follows to adapt the T2Bio existing assay workflow and nucleic acid detection methodology for detection of $B$. thuringiensis. In summary, the following steps were followed:

1. Primer and capture probes were designed \& synthesized

2. Base reagents were obtained (Bt genomic DNA, viable Bt cultures, Charles River water)

3. Particles were functionalized with the oligonucleotide hybridization probes

4. Multiple amplification enzymes and conditions were screened for amplification efficiency in buffer and river water

5. PCR method was optimized on genomic Bt DNA and Bt cells

6. Particle hybridization and clustering with the amplified PCR product was optimized

7. Sample matrix effects $\&$ robustness was demonstrated

8. Assay processing steps, ( e.g. up-front sample concentration, lysis, etc) were tested and optimized

9. Complete demonstration of Bt in river water on final T2Bio instrumentation (NG MR reader and HTP system)

All of this work occurred on the different versions of the MR reader as well as the HTP system. Both semi-automated and fully manual assay processing methods were used during assay development. As will be shown, the final assay was capable of detecting $10 \mathrm{CFU} / \mathrm{mL}$ of $\mathrm{B}$. thuringiensis directly within an environmental water sample (i.e. river water) within 90 minutes. 
Final Scientific \& Technical Report: Development of a System for Rapid Detection of Contaminants in Water Supplies Using Magnetic Resonance and Nanoparticles

\section{Reagent Design and Development}

A set of PCR primers were designed which recognize and amplify the $B$. thuringiensis servoar kurstaki (BGSC-4D12) soluble lytic murein transglyosidase (slt) locus within the bacterial genome (GenBank EF595776.1). The primers were designed to create an amplified product (amplicon) with a length of approximately 200 bases. Additionally, the primers were specifically designed for asymmetric PCR to create an excess of single stranded product. Single stranded product more readily hybridizes and facilitates particle clustering. To design the primers for asymmetric PCR, the melting temperatures were adjusted for primer concentrations by designing them to have different melting temperatures at their respective concentrations (a difference in $T_{m}$ of $\sim 5^{\circ} \mathrm{C}$ ). The $T_{m}$ of the forward primer was $60^{\circ} \mathrm{C}$ at a concentration of $300 \mathrm{nM}$ while that of the reverse primer was $55^{\circ} \mathrm{C}$ at a concentration of $75 \mathrm{nM}$. The divalent cation concentration was $3 \mathrm{mM}$ and the monovalent cation was kept at the default of $50 \mathrm{mM}$.

The capture probes were designed to hybridize in a nested fashion to the PCR amplicon. Having nested sequences for the primers and capture probes provides an additional screen for specificity of the overall assay. The capture probes were designed as synthetic oligonucleotides that were covalently attached to superparamagnetic particles. Due to their central role in facilitating particle clustering, their design relative to the amplicon is critical for realizing optimal performance. Accordingly, steric hindrance of the particles was minimized by positioning the capture probes at either end of the strand of DNA. The capture probe that recognized and bound to the $5^{\prime}$ end of the amplicon was $3^{\prime}$ aminated while the capture probe that recognized and bound to the $3^{\prime}$ end of the amplicon was $5^{\prime}$ aminated. The probes were designed to have melting temperatures of $70^{\circ} \mathrm{C}$ with the monovalent cation concentration adjusted to $300 \mathrm{mM}$. The sequences of the forward and reverse primers and the particle bound capture probes are shown in Figure 22. PCR primers and probes were procurred from IDT Technologies (Coralville, IA). All primers and probes were purified via HPLC.

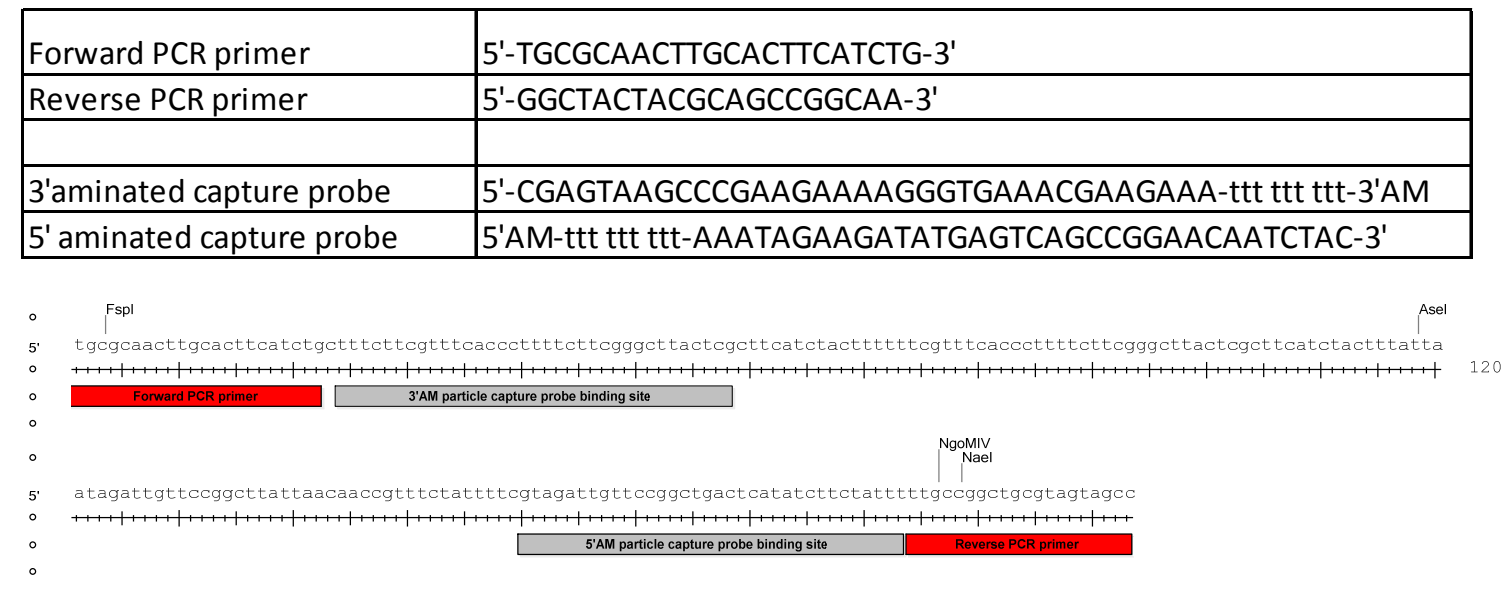

Figure 22. Sequences of $B$. thuringiensis PCR primers and particle bound capture probes and their location upon hybrization to the sense strand of the B. thuringiensis slt operon. PCR primers were chemically unmodified. The particle capture probes were aminated (AM) at their $3^{\prime}$ end 5' ends, respectively. A poly-T linker(T9) was incorporated between the amine and the last DNA base of the probe to serve as a linker promoting complete T2 Biosystems 
Final Scientific \& Technical Report: Development of a System for Rapid Detection of Contaminants in Water Supplies Using Magnetic Resonance and Nanoparticles

display of the probe and minimizing probe/particle interactions. Capture particle binding sites were nested within the PCR amplicon.

Each of the aminated capture probes were covalently conjugated to 1 micron carboxylated superparamagnetic particles (T2 Biosystems, Cambridge MA) using standard EDC chemistry. Two pools of particles were generated, one bearing the $5^{\prime}$ aminated and one bearing the $3^{\prime}$ aminated probes respectively. Particle amount per reaction was standardized based on the concentration of iron per assay. Functional oligonucleotide graft densities were measured by hybridizing radiolabeled complements to particles, washing the particles $3 \mathrm{X}$ with $\mathrm{TE}$, eluting the hybridized targets via heat denaturation and quantifying the bound DNA via scintillation counts.

Due to the goal of conducting PCR within environmental water samples, polymerases were identified that can tolerate some of the common inhibitors found within river or pond water (i.e. humic acid). Three commercially available enzymes were tested: HemoKlenTaq (NEB, Ipswich, MA), Phusion (Finnzymes) and Phire Direct Plant (Finnzymes).

B. thuringiensis serovar kurstaki (BGSC-4D12) cells and isolated genomic DNA were procured from the Bacillus Genome Stock Center (Columbus, $\mathrm{OH}$ ). Bacterial cells were cultivated on Luria Bertani agar plates at room temperature (nominally $\sim 25^{\circ} \mathrm{C}$ ). Spiked samples were prepared from an isolated $\mathrm{Bt}$ colony that was selected from LB plates. Cells were washed three times with PBS prior to counting using a hemocytometer. Serial dilutions of cells were prepared using standard dilution methods. The environmental water sample (>1 L) was obtained from the Charles River in the fall of 2009. The sample was not subjected to any filtration or purification and as such contained large amounts of sediment and organic detritus. The river water sample was stored at $-80^{\circ} \mathrm{C}$ and thawed prior to use.

\section{Detection of Bacillus thuringiensis in buffer}

The initial step in assay development was establishing a baseline performance for the designed primers, probes, PCR reaction conditions, and detection particles. Accordingly, a PCR reaction was conducted using the Phire Direct Plant enzyme on ten-fold serial dilution of cells or genomic DNA in a 1x PBS buffer solution. The results of these initial reactions are shown in Figure 23. Details of the PCR reaction composition, thermocycling protocol, and hybridization mediated agglomeration reactions are provided in the legend of Figure 23.

As shown in Figure 23, a change in the measured $\mathrm{T}_{2}$ values of $~ 600 \mathrm{msec}$ at 10 copies of genomic DNA/reaction and $\sim 700 \mathrm{msec}$ at 1000 cells/reaction was observed. These are substantial changes in measured $T_{2}$ values for a dilute amount of target. However, it was observed that the measured $T_{2}$ values reduced with increasing amount of target as concentrations increased from 10 copies/reaction or 1000 cells/reaction. This reduction in measured $T_{2}$ values was hypothesized to arise from a "prozone" affect, or an inhibition of particle clustering due to an over-titration of the particles with excess target. In other words, when a particle agglutination reaction is overtitrated with excess target, all surface-immobilized capture probes are saturated with target leaving no probes available to participate in a particle-to-

T2 Biosystems

DOE project DE-SC0001821

Page 29 of 38 
Final Scientific \& Technical Report: Development of a System for Rapid Detection of Contaminants in Water Supplies Using Magnetic Resonance and Nanoparticles

particle cross-linking event by hybridizing to an amplicon bound by another capture probe and particle. To confirm whether the reduced switch at high target levels was due to prozone, the amplicon generated from the genomic DNA template was diluted and subjected to particle agglutination and $T_{2}$ measurement. For this data, a linear response from 100 copies to 10,000 copies/reaction was observed after 10 -fold dilution of the amplification reaction. Additionally, a loss of signal at 10 copies/reaction was also observed. Both of these observations from Figure 23b confirmed that excess amplified target lead to a prozoned signal in Figure 23a.



Figure 23. Asymmetric PCR amplification and subsequent $\mathrm{T}_{2}$ detection of amplicon-induced particle agglomeration. PCR reactions were conducted with serially diluted $B$. thuringiensis cells or genomic DNA using the Phire Direct Plant polymerase. The reactions contained 1X Phire Plant PCR buffer, $300 \mathrm{nM}$ of the forward PCR primer, $75 \mathrm{nM}$ of the reverse PCR primer and $1 \mathrm{uL}$ of the Phire polymerase in a $50 \mathrm{uL}$ reaction volume. The reactions were subjected to an initial 5 minute denaturation at $98^{\circ} \mathrm{C}, 40$ cycles of a denaturation at $98^{\circ} \mathrm{C}$ for 5 seconds, annealing at $55^{\circ} \mathrm{C}$ for 5 seconds, extension at $72^{\circ} \mathrm{C}$ for 20 seconds, and 1 minute of a final extension at $72^{\circ} \mathrm{C}$. Post-PCR, $10 \mathrm{uL}$ of amplicon was removed and mixed with $5 \mathrm{uL}$ of oligonucleotide derivatized superparamagnetic nanoparticles, at an iron concentration of $1 \mathrm{mM}$, suspended in 6XSSC and 30\% formamide. The reactions were denatured for 3 minutes at $95^{\circ} \mathrm{C}$ and then incubated for 30 minutes at $60^{\circ} \mathrm{C}$ in a shaker incubator agitating at a speed of $1000 \mathrm{rpm}$ (Vortemp, LabSystems). The reactions were then diluted with $135 \mathrm{uL}$ of PBS, $0.1 \%$ BSA, $0.1 \%$ Tween-20 and incubated for 10 minutes in an hMAA magnet at $37^{\circ} \mathrm{C}$. The samples were then subjected to a brief vortex, 1 minute temperature equilibration at $37^{\circ} \mathrm{C}$, and $a T_{2}$ measurement. (a) A scatter plot of the $T_{2}$ readings on the samples processed as described; (b) a scatter plot of the samples processed with an additional ten-fold dilution of the post-PCR sample prior to addition to the particles.

These initial experiments demonstrated that the assay reagents were generally working as intended. Although the limit of detection of 1000 cells/reaction was several orders of magnitude from the targeted limit of detection of $10 \mathrm{CFU} / \mathrm{mL}$, and the sample matrix was buffer not river water, this basic assay formulation served as a starting point for subsequent assay development.

\section{Detection of Bt genomic DNA in an Environmental Water Sample}

To test the robustness of the assay workflow and amplification enzyme to an environmental sample, PCR reactions were conducted on genomic DNA spiked into nuclease-fee de-ionized water (Ambion, Austin, TX) and an aliquot of the Charles river water sample. The data indicated that there was no inhibition of the activity of the Phire Direct Plant polymerase within the river water (Figure 24). 
Final Scientific \& Technical Report: Development of a System for Rapid Detection of Contaminants in Water Supplies Using Magnetic Resonance and Nanoparticles

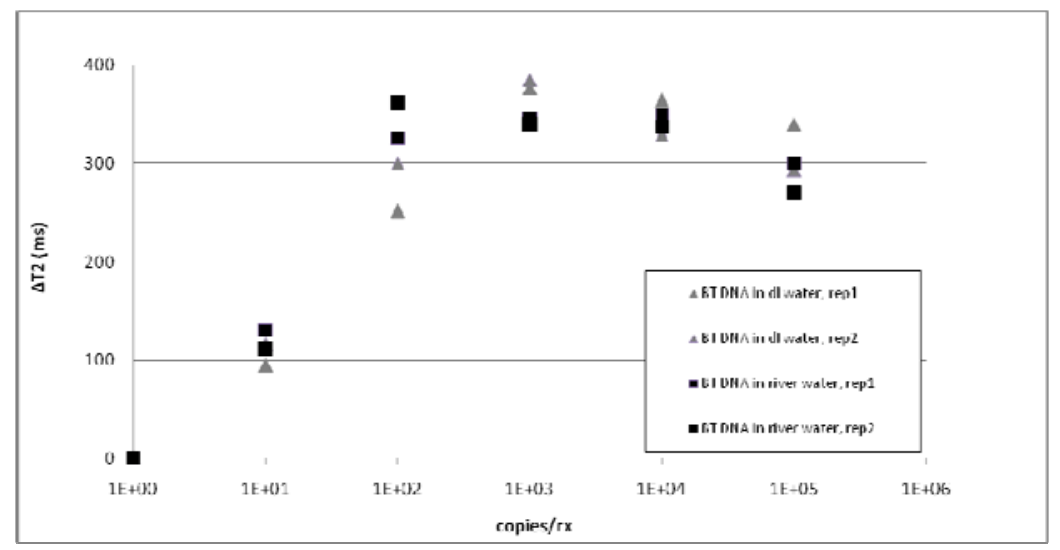

Figure 24: Detection of $B$. thuringiensis genomic DNA in di-ionized water and Charles River water. Purified genomic DNA was serially diluted in PBS. Approximately 5 uL of the diluted genomic DNA was then spiked into 20 $\mathrm{uL}$ of either de-ionized water or Charles River water. PCR reactions were conducted using the entire $20 \mathrm{uL}$ sample as indicated in Figure23. Detection reactions were also conducted as indicated in Figure 23 except $5 \mathrm{uL}$ of the amplicon was incubated with the particles instead of $10 \mathrm{uL}$.

The data in Figure 24 supports the conclusion that the overall assay workflow was agnostic to purified or dirty river water. This is not typical to conventional nucleic acid detection methodologies. Typically both the amplification and detection steps are prone to interference from substances in dirty samples such as river water.

\section{Development and implementation of an assay work-flow that increases Bt detection sensitivity}

Although the assay reagents and workflow robustly performs in the target matrix, the assay sensitivity of $\sim 1000$ cells/reaction is $>10,000 x$ worse than the target sensitivity of $10 \mathrm{CFU} / \mathrm{mL}$. The workflow that was used to collect the data in Figures $23 \& 24$ basically consisted of the following steps: (1) direct addition of the sample to the PCR reaction; (2) heat lysis; (3) amplification; (4) incubation with the superparamagnetic nanoparticles; and (5) $\mathrm{T}_{2}$ measurement. This assay workflow did not utilize any upfront enrichment and was utilizing heat lysis to lyse the target cells, which may have yielded incomplete lysis.

To improve the overall assay sensitivity, two changes to the overall assay workflow were made. An upfront sample enrichment step was added followed by a mechanical lysis approach. This assay process was based on previous data generated at T2Bio (Figure 25) demonstrating fungal pathogenic organisms could be sensitively detected in whole blood. In this workflow, the target fungal cells were concentrated from human whole blood via centrifugation at $6000 \mathrm{~g}$ for 5 minutes. Further, when this cell pellet was subjected to bead beating using a standard laboratory vortexer and silica glass beads, sufficient quantities of intact fungal DNA were liberated in a reproducible fashion to enable highly sensitive detection directly in the dirty sample. Because fungal targets are particularly difficult cells to lyse, it was decided to adopt this workflow in the Bt assay and determine if it too afforded increased detection sensitivity. 


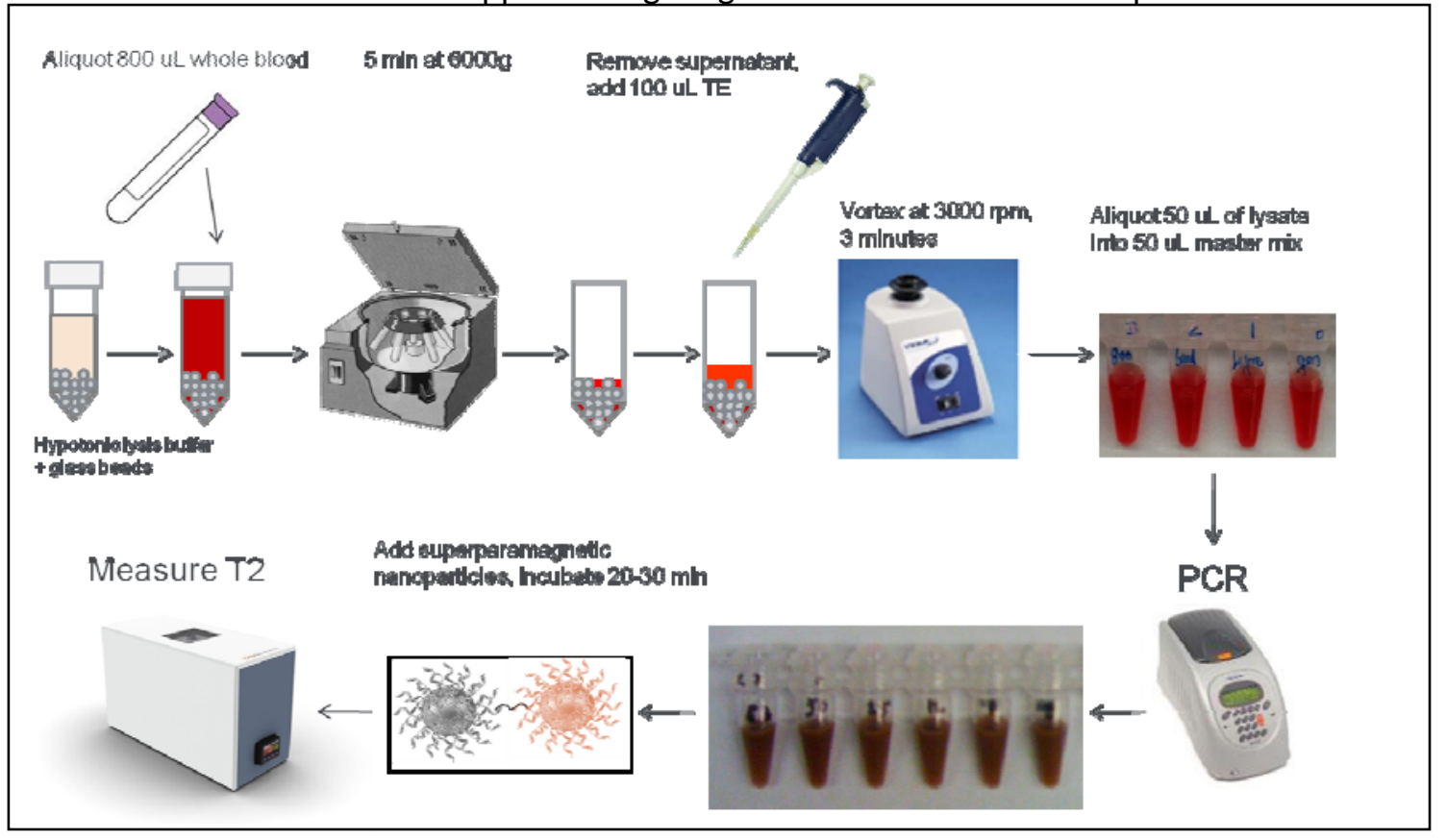

Figure 25: Outline of the whole blood fungal workflow upon which the Bt detection workflow was based. Within this workflow $0.8 \mathrm{~mL}$ to $1 \mathrm{~mL}$ of human whole blood was added to a reaction tube containing a hypotonic lysis solution and acid washed glass beads. The lysed blood was then subjected to a 5 minute centrifugation at 6,000 G. After removal of the supernatant, $100 \mathrm{uL}$ of TE was added and the sample was subjected to vortexing at 3,000 rpm for 5 minutes to lyse the target cells. 50 uL cell lysate was added to $50 \mathrm{uL}$ of PCR master mix containing a whole blood compatible polymerase. Following amplification, $10 \mathrm{uL}$ of the PCR reaction was added to $5 \mathrm{uL}$ of capture probe derivatized superparamagnetic nanoparticles. The detection reactions are then conducted as indicated in Figure 23.

The assay workflow described in Figure 25 was applied to detection of Bt without using the hypotonic lysis step. An experiment was conducted to determine the separate contributions of the up-front enrichment process and mechanical lysis on overall assay sensitivity. Accordingly, two replicates of serially diluted Bt cells in $1 \mathrm{~mL}$ of water were prepared. Both replicates were subjected to a 6,000 G centrifugation, decanted, and hydrated with $100 \mathrm{uL}$ of TE. Only one replicate was subjected to mechanical lysis by adding $125 \mathrm{mg}$ of acid washed $0.5 \mathrm{~mm}$ glass beads and vortexing at 3,000 rpm for 5 minutes prior to addition of $50 \mathrm{uL}$ to the PCR master mix. The other replicate was not subjected to mechanical lysis, but rather was merely resuspended and a $50 \mathrm{uL}$ aliquot was added directly to the PCR master mix. In this case, the Phire amplification enzyme was used. As shown in Figure 26, a reproducible detection sensitivity of 10 cells $/ \mathrm{mL}$ was achieved using up-front enrichment and mechanical lysis while a detection sensitivity of $\sim 100$ cells $/ \mathrm{mL}$ was achived with the standard heat lysis method. Therefore, heat lysis had a $10 \%$ yield as compared to the lysis yield of mechanical lysis. Other data on fungal cells shows that mechanical lysis has $\sim 100 \%$ efficiency under these conditions. 
Final Scientific \& Technical Report: Development of a System for Rapid Detection of Contaminants in Water Supplies Using Magnetic Resonance and Nanoparticles

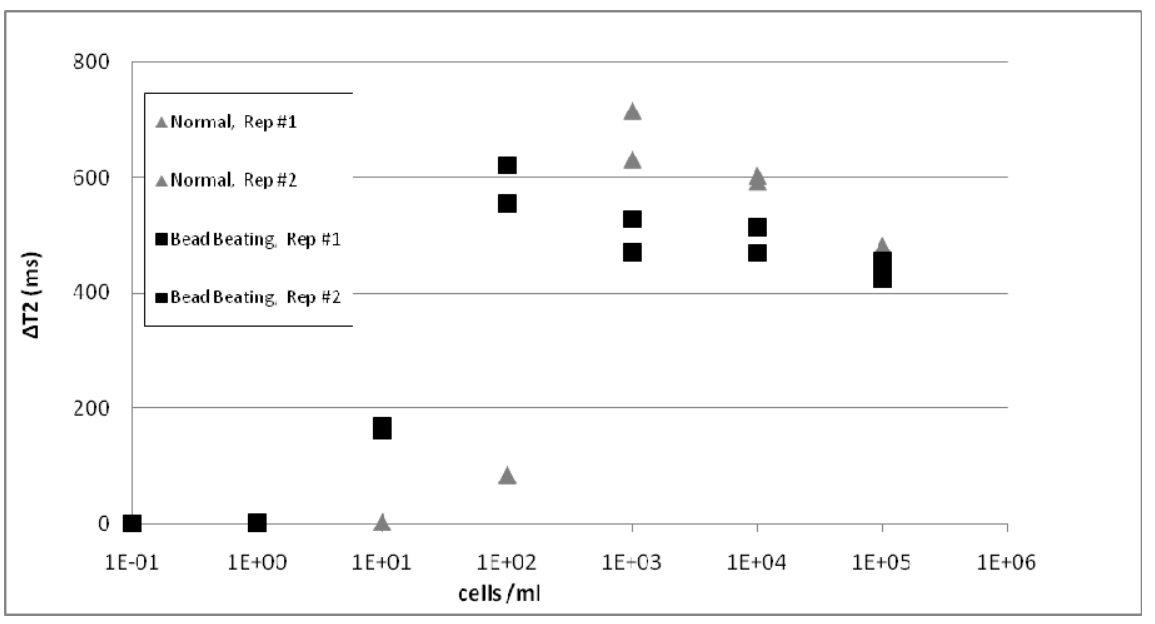

Figure 26: Addition of enrichment and mechanical lysis steps improved the $T_{2}$ detection sensitivity over a standard enrichment/heat lysis protocol by several orders of magnitude. The LOD for the new workflow using upfront enrichment and mechanical lysis was 10 cells $/ \mathrm{mL}$ while the LOD for a up-front enrichment and the standard heat lysis was 100 cells $/ \mathrm{mL}$.

The data in Figure 26, where the detection sensitivity was 10 cells $/ \mathrm{mL}$, compared to the data in Figures $23 \& 24$, where the detection sensitivity was $\sim 10,000$ cells $/ \mathrm{mL}$ ( $\sim 1000$ cells/reaction for a $\sim 100 \mathrm{uL}$ reaction), indicates that the upfront enrichment step provided a boost in sensitivity of $\sim 100 \mathrm{x}$ and the mechanical lysis step provided a boost in sensitivity of 10x.

In summary, the data shown in Figure 26 demonstrated that the new work-flow could increase the detection sensitivity to within range of the desired sensitivity of $10 \mathrm{CFU} / \mathrm{mL}$. However, this was with pure water. One potential limitation of the up-front enrichment step could be if the concentrated river water sample compromised the amplification efficiency due to concentrated inhibitory sediment, organic compounds, and detritus, and thereby led to a diminished detection sensitivity.

A series of optimization experiments were conducted to determine the conditions under which a reproducible and sensitive $T_{2}$ dose response curve could be obtained. One example data set is shown in Figure 27 where the $T_{2}$ measurements were obtained using the high throughput system. The significant advantage of the high throughput system for assay development was that large sets of samples could be prepared and loaded, to be subsequently run in a hands-free fashion. This greatly aided in increasing the bandwidth of the existing assay development team and also allowed for large data sets to be obtained in a reliable, reproducible manner. 
Final Scientific \& Technical Report: Development of a System for Rapid Detection of Contaminants in Water Supplies Using Magnetic Resonance and Nanoparticles

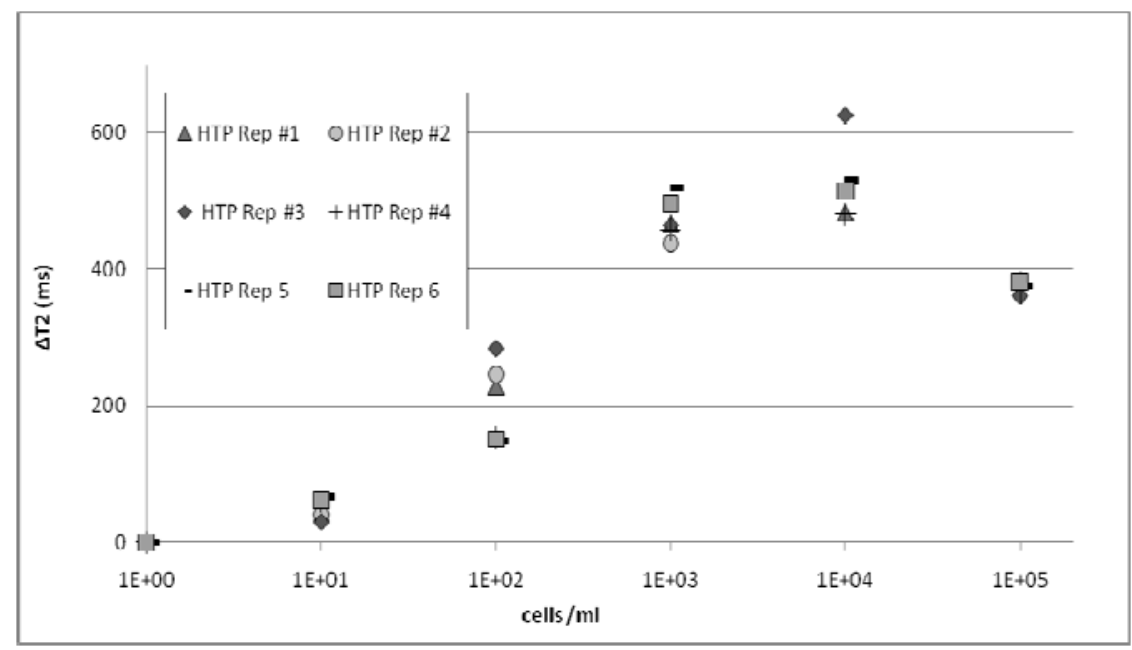

Figure 27. Sample data set from the high throughput system (HTP). B. thuringiensis cells were serially diluted, enriched, and mechanically lysed as outlined in Figure 25. In this study, two replicate PCR reactions were conducted per dilution and 3 detection reactions were subsequently set up from each PCR reaction yielding an $\mathrm{n}=6$. The $\mathrm{PCR}$ and detection reactions were conducted as indicated in Figure 25 except the diluted detection reactions were loaded onto the HTP system which automates the hMAA, vortex, incubation, and $T_{2}$ detection steps.

\section{Detection of Bt cells within environmental water samples}

To determine the affect of river water on the assay performance, spikes of $B$. thuringiensis cells in Charles River water were prepared as described above and the samples were subjected to the workflow shown in Figure 25. As shown in Figure 28, a similar detection sensitivity of $10 \mathrm{CFU} / \mathrm{mL}$ was obtained in river water, demonstrating that the change in sample matrix had no impact on the overall performance of the assay.

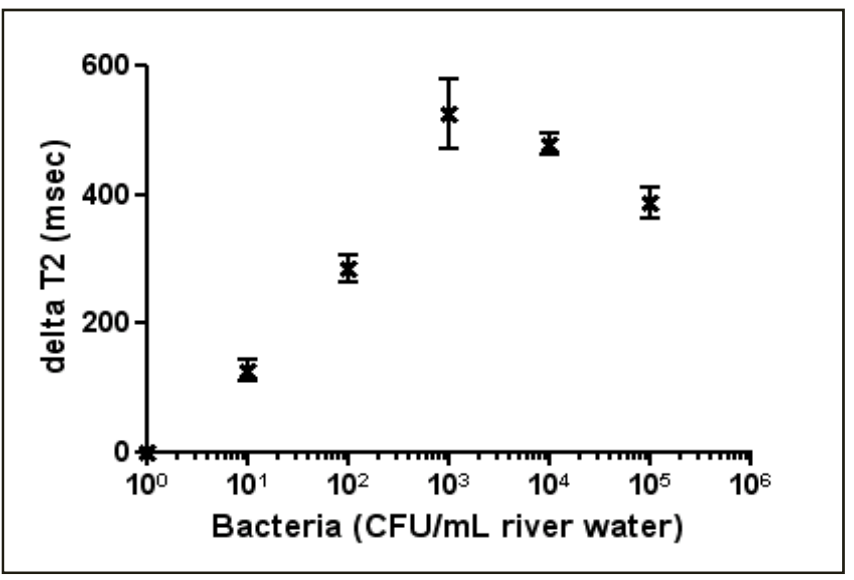

Figure 28. $T_{2}$ detection of $B$. thuringiensis in Charles River water. The limit of detection was observed to be 10 $\mathrm{CFU} / \mathrm{mL}$. One milliliter of Charles River water was spiked with $10 \mathrm{uL}$ of TE containing serially diluted $B$.

T2 Biosystems 
Final Scientific \& Technical Report: Development of a System for Rapid Detection of Contaminants in Water Supplies Using Magnetic Resonance and Nanoparticles

thuringiensis cells. $125 \mathrm{mg}$ of acid washed glass beads were added to each tube and they were subjected to centrifugation at $6000 \mathrm{G}$ for 5 minutes. The supernatant was removed and $100 \mathrm{uL}$ of TE was added to the bead matrix. The samples were vortexed at $3000 \mathrm{rpm}$ for 5 minutes and $50 \mathrm{uL}$ of the lysate was included in a PCR reaction containing $50 \mathrm{uL}$ of Phire Direct Plan polymerase master mix.

\section{Detection of Bt cells within a human biological sample matrix}

The matrix compatibility of the $B$. thuringiensis detection assay was further demonstrated by running measurements in human whole blood. Other work at T2Bio had demonstrated similar performance for detection of a fungal pathogen within human whole blood. To accommodate the different sample matrix, the polymerase was changed from the Phire Direct Plant (Finnzymes) to HemoKlenTaq (NEB) and Phusion (Finnzymes). Figure 29 shows the results for both of these enzymes, where both detected a concentration of $10 \mathrm{CFU} / \mathrm{mL}$ of $B$. thuringiensis directly within human whole blood. These results further demonstrate the adaptability, robustness, and sensitivity of the T2Bio molecular diagnostics assay across different sample matrices.

(a)

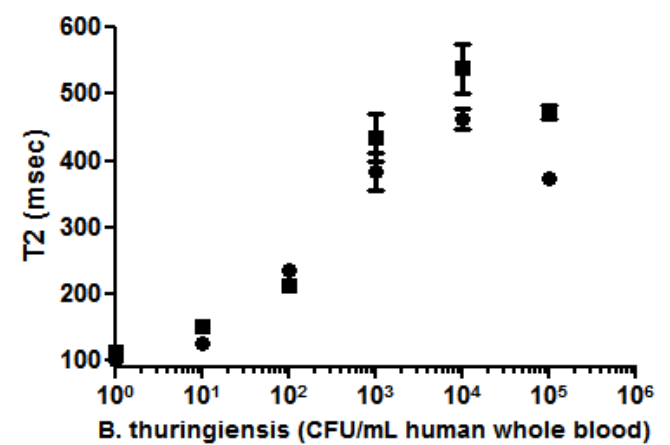

(b)

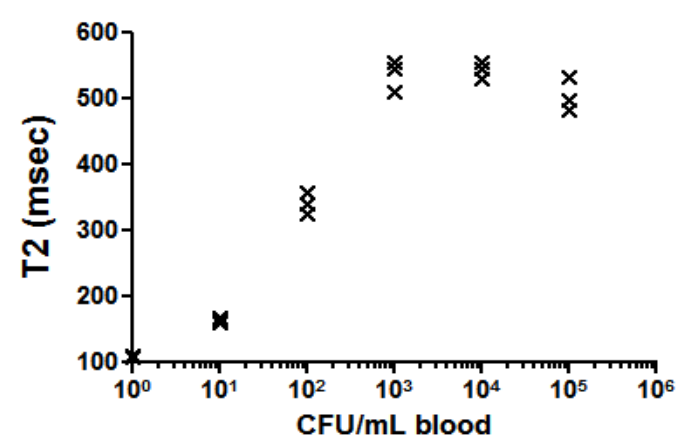

Figure 29. T2-based detection of $B$. thuringiensis cells spiked into human whole blood. One milliliter aliquots of healthy donor blood (fresh human blood drawn in $\mathrm{K}_{2}$ EDTA plastic vacutainer vials) were spiked with serially diluted $B$. thuringiensis cells. The up-front work-flow as well as the T2 detection reactions were conducted as outlined in Figure 25. (a) A scatterplot of the results obtained with the HemoKlentaq enzyme. Fifty microliters of lysate was added to a HemoKlentaq master mix such that upon dilution the reaction composition would be: 1x HemoKlentaq reaction buffer, 10 mM dNTPs, 300 nM forward PCR primer, 75 nM PCR reverse primer, and $10 \mathrm{uL}$ of HemoKlentaq polymerase. The cycling profile included an initial denaturation at $95^{\circ} \mathrm{C}$ for 3 minutes, 40 cycles of a 20 second denaturation at $95^{\circ} \mathrm{C}, 30$ second annealing at $55^{\circ} \mathrm{C}, 20$ second elongation at $68^{\circ} \mathrm{C}$, and a ten minute final extension at $68^{\circ} \mathrm{C}$. (b) A scatterplot of the results obtained with Phusion polymerase. Fifty microliters of lysate was added to $50 \mathrm{uL}$ of Phusion master mix which upon dilution yields a reaction comprised of $1 \mathrm{X}$ Phusion buffer, $300 \mathrm{nM}$ Forward PCR primer, $75 \mathrm{nM}$ Reverse PCR primer, dNTPs, and 2 uL of Phusion polymerase. The cycling parameters are identical to that used in Figure 23. 
Final Scientific \& Technical Report: Development of a System for Rapid Detection of Contaminants in Water Supplies Using Magnetic Resonance and Nanoparticles

\section{Determining Assay Specificity}

The data shown above demonstrates a robust and sensitive assay for the detection of $B$. thuringiensis within an environmental water sample and also within a crude biological matrix. Additional work was completed to explore the specificity of the assay for $B$. thuringiensis. As such, a closely related organism, $B$. cereus, was selected to challenge the specificity of the assay. As discussed above, the genomes of $B$. thuringiensis and $B$. cereus have a high degree of sequence identity, as shown in Figure 30.

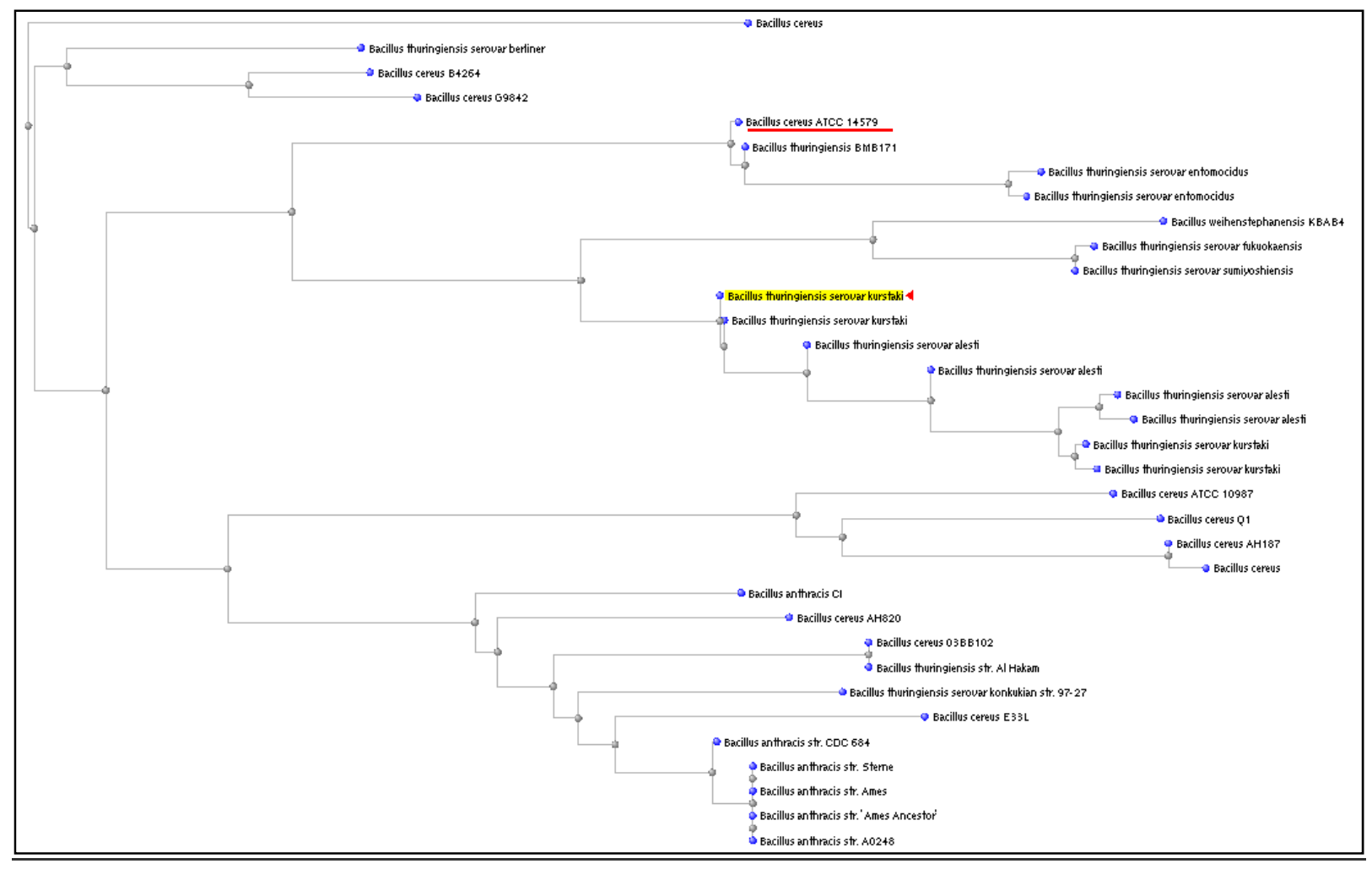

Figure 30. Neighbor joining phylogenetic distance tree illustrating the relationship of 34 species from the Bacillus genus. B. thuringiensis serovar kurstaki is highlighted in yellow. The B. cereus strain ATCC 14579 which was used in the specificity study is underlined in red.

A sequence alignment conducted between $B$. thuringiensis indicated that the PCR primer binding sites, as depicted by the arrows in Figure 31, were conserved between $B$. thuringiensis BGSC-4D12 and $B$. cereus ATCC 14579. The capture probe binding sites had two base changes per capture probe binding site (shown in blue in Figure 31). It is noteworthy that one of these base changes within each capture probe binding site would form a G-T base pair upon hybridization of the probe (as illustrated with a "star" in Figure 31). This is a particularly permissive mismatch and is extremely difficult to discriminate via simple hybridization of a probe comprised of standard DNA nucleotides (Allawi H.T. 1997). A 10-fold serial dilution of both the $B$. thuriengensis and $B$. cereus genomic DNA was conducted. The amplification reactions were conducted and gel electrophoresis confirmed that for both species an amplicon of identical size was formed and similar amplification yields was obtained (data not shown). The amplicons 
Final Scientific \& Technical Report: Development of a System for Rapid Detection of Contaminants in Water Supplies Using Magnetic Resonance and Nanoparticles

from each reaction were incubated with $B$. thuringiensis-specific superparamagnetic nanoparticles to obtain the dose response curves in Figure 31. Interestingly, a 3-fold reduction in $T_{2}$ signal was observed for detection of the $B$. cereus amplicon with the $B$. thuringiensis particles. These results indicated that the $T_{2}$ particle clustering assay is not only sensitive to presence of the intended target but also that it can be used to discriminate target detection from closely related organisms. Additional optimization of hybridization conditions could certainly lead to an even greater degree of specificity.
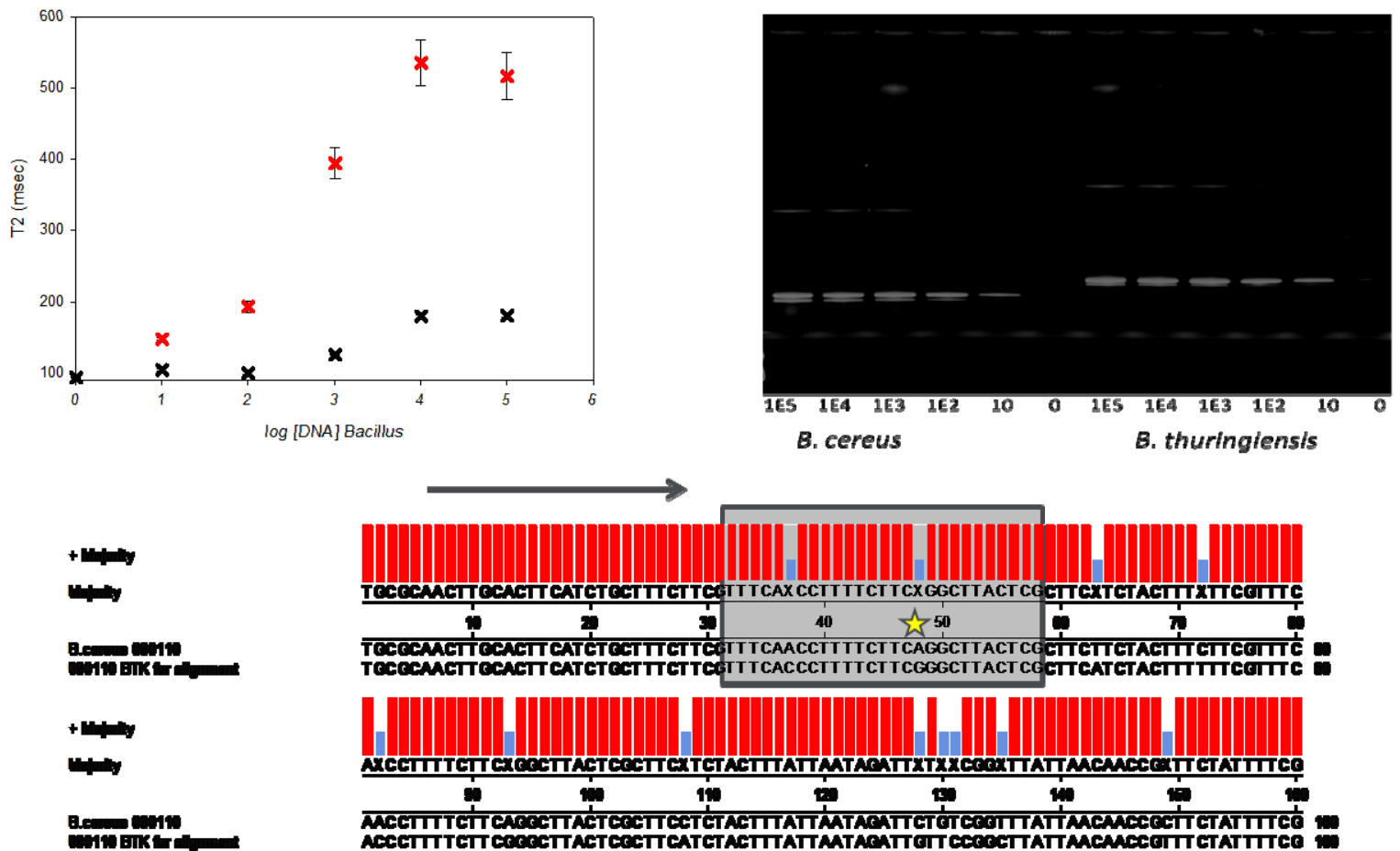

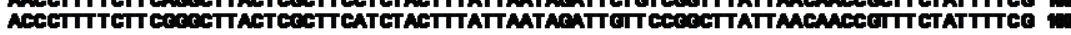


Figure 31. Discrimination of two closely related species of Bacilli. The $T_{2}$ measurements conducted using amplified $B$. cereus or $B$. thuringiensis DNA are shown in the scatter plot on the top. The DNA sequence alignment of the two species is shown on the bottom. Both Bacilli were amplified using the same PCR primers (arrows in sequence alignment) that have perfect homology to either species. The detection reactions were conducted using particles bearing capture probes specific to Bacillus thuringiensis (gray boxes). All base changes are shown in blue and the GT wobbles that form upon hybridization are indicated with yellow stars. Greater than 3-fold discrimination between $B$. cereus and $B$. thuringiensis at a concentrations spanning $10 \mathrm{CFU} / \mathrm{mL}$ to $10 \mathrm{E} 5 \mathrm{CFU} / \mathrm{mL}$ was observed. 
Final Scientific \& Technical Report: Development of a System for Rapid Detection of Contaminants in Water Supplies Using Magnetic Resonance and Nanoparticles

\subsection{Products developed under the award}

The data, instruments, and equipment described above summarizes the instrumentation and assay architecture that was developed under this award. Rapid and portable detection of bioterrorism agents and a broad range of health and environmental threats in contaminated water will prevent the spread of disease throughout our communities, enabling public health officials to take swift action to prevent the loss of lives. With a platform that can be deployed at virtually any site of interest, information can be obtained quickly to determine the status and potential impact of dangerous pathogens. Further, the completion of this project has resulted in advances in the application of nanotechnology to diagnostics, biosensing, and patient care. This funded project is a direct example of the DOE's mission to promote scientific and technological innovation in support of advancing the national, economic, and energy security of the United States. Of the strategic themes set by the Department to achieve this mission, T2 Biosystems's work aligns directly with the third: Scientific Discovery and Innovation: strengthening U.S. scientific discovery, economic competitiveness, and improving quality of life through innovations in science and technology. As described, the product of this award was application of the T2 Biosystems assay technology to pathogen detection in an environmental sample. No publications or other public release of the results were made. No patent applications describing the assays or the data from these funded assays were filed as a result of this work.

\subsection{References}

Allawi H.T., a. J. S., Jr. Thermodynamics and NMR of Internal GâT Mismatches in DNA. Biochemistry 1997 36: 10581-10594.

Erlendur Helgason, O. A. Ø., Dominique A. Caugant, Henning A. Johansen, Agnes Fouet, Michéle Mock, Ida Hegna, and Anne-Brit Kolst $\varnothing$. Bacillus anthracis, Bacillus cereus, and Bacillus thuringiensis-One Species on the Basis of Genetic Evidence. Applied and Environmental Microbiology 2000 66(6): 26272630.

Lowery TJ. Nanomaterials-Based Magnetic Relaxation Biosensors. In: Kumar, CSSR, ed. Nanomaterials for the Life Sciences Vol. 4: Magnetic Nanomaterials. Weinheim: Wiley-VCH Verlag GmbH \& Co. KGaA, 2009: 3-53.

Vilas-Bôas GT, P. A., Arantes OM. Biology and taxonomy of Bacillus cereus, Bacillus anthracis, and Bacillus thuringiensis. Can J Microbiol. 2007 53(6): 673-687. 